\title{
Some Properties of Distances and Best Proximity Points of Cyclic Proximal Contractions in Metric Spaces
}

\author{
M. De La Sen ${ }^{1}$ and Asier Ibeas ${ }^{2}$ \\ ${ }^{1}$ Institute of Research and Development of Processes, Campus of Leioa (Bizkaia), University of the Basque Country, \\ P.O. Box 644 Bilbao, Barrio Sarriena, 48940 Leioa, Spain \\ ${ }^{2}$ Department of Telecommunications and Systems Engineering, Autonomous University of Barcelona, Bellaterra, \\ 08193 Barcelona, Spain \\ Correspondence should be addressed to M. De La Sen; manuel.delasen@ehu.es
}

Received 9 May 2014; Revised 22 July 2014; Accepted 5 August 2014; Published 15 October 2014

Academic Editor: Adrian Petrusel

Copyright (c) 2014 M. De La Sen and A. Ibeas. This is an open access article distributed under the Creative Commons Attribution License, which permits unrestricted use, distribution, and reproduction in any medium, provided the original work is properly cited.

This paper presents some results concerning the properties of distances and existence and uniqueness of best proximity points of $p$-cyclic proximal, weak proximal contractions, and some of their generalizations for the non-self-mapping $T: \bigcup_{i \in \bar{p}} A_{i} \rightarrow$ $\bigcup_{i \in \bar{p}} B_{i}(p \geq 2)$, where $A_{i}$ and $B_{i}, \forall i \in \bar{p}=\{1,2, \ldots, p\}$, are nonempty subsets of $X$ which satisfy $T\left(A_{i}\right) \subseteq B_{i}, \forall i \in \bar{p}$, such that $(X, d)$ is a metric space. The boundedness and the convergence of the sequences of distances in the domains and in their respective image sets of the cyclic proximal and weak cyclic proximal non-self-mapping, and of some of their generalizations are investigated. The existence and uniqueness of the best proximity points and the properties of convergence of the iterates to such points are also addressed.

\section{Introduction}

The characterization and study of existence and uniqueness of best proximity points is an important tool in fixed point theory concerning cyclic nonexpansive mappings including the problems of (strict) contractions, asymptotic contractions, contractive, weak-contractive mappings, and cyclic mappings and also in related problems of proximal contractions, weak proximal contractions, and approximation results and methods [1-15]. The application of the theory of fixed points in stability issues of dynamic systems, [16-21] has been proved to be a very useful tool. See, for instance, [22-26] and references therein. Some best approximation problems in semiconvex and locally convex structures and HyersUlam type stability in multivalued functions and in additivequadratic functional equations are investigated in [27-30] and some of the references therein. Recent trends concerning best proximity points and related problems are dealt with in [31-35] and some references therein. In particular, the problem of best proximity points of two mappings in a cyclic disposal is investigated in [31] under a nonlinear contractive condition. In [32], several results are obtained for proximal and weak proximal contractions of several types as well as for generalized proximal nonexpansive mappings. A modified Suzuki $\alpha-\psi$ proximal contraction is proposed and discussed in [33] and "ad hoc" best proximity and fixed point results are obtained. Generalizations of proximal contractions of first and second kinds are given in $[34,35]$ for non-self-mappings and related optimal approximate solution theorems are obtained.

This paper is devoted to formulating and proving some results being concerned with the boundedness and convergence properties of distances and the convergence of the built iterated sequences to unique existing best proximity points of $p$-cyclic proximal and weak proximal contractions of the form $T: \bigcup_{i \in \bar{p}} A_{i} \rightarrow \bigcup_{i \in \bar{p}} B_{i}(p \geq 2)$ where $A_{i}$ and $B_{i}$, for all $i \in \bar{p}=\{1,2, \ldots, p\}$, are nonempty subsets of $X$ which satisfy $T\left(A_{i}\right) \subseteq B_{i}$, for all $i \in \bar{p}$, with $(X, d)$ being a metric space. In the most general case, all the $A_{i}$ and $B_{i}$ pairs of subsets, for all $i \in \bar{p}$, are assumed to be pairwise disjoint. 
The results are also extended to a class of generalized $p$ cyclic proximal and weak proximal contractions in the sense that the contractiveness constraints are referred to finite sets of consecutive iterations rather than to each iteration. The boundedness and convergence of the sequences of distances in the domains and image sets of the cyclic proximal and weak cyclic proximal non-self-mappings are investigated. The existence and uniqueness of the best proximity points and their allocation as limit points, or limit cycles of best proximity points, are also addressed. These last properties are achieved if the metric space is complete under approximative compactness' assumptions of the image subsets of the cyclic mapping with respect to the domain subsets.

\section{2. p-Cyclic Proximal Contractions, Extensions, Boundedness, and Convergence of Distances}

Consider the metric space $(X, d)$ and subsets $A_{i}$ and $B_{i}$ of $X$ for $i \in \bar{p}$, where $\bar{p}=\{1,2, \ldots, p\}$ with $p \geq 2$. Consider also a non-self-mapping $T: \bigcup_{i \in \bar{p}} A_{i} \rightarrow \bigcup_{i \in \bar{p}} B_{i}$, satisfying $T\left(A_{i}\right) \subseteq B_{i}$, for all $i \in \bar{p}$. Assume that $D_{i}=d\left(A_{i+1}, B_{i}\right), D_{A}=$ $d\left(A_{i}, A_{i+1}\right)$, and $D_{B}=d\left(B_{i}, B_{i+1}\right)$, for all $i \in \bar{p}$ by assuming also that $A_{n p+i}=A_{i}$ and $B_{n p+i}=B_{i}$, for all $i \in \bar{p}$, for all $n \in$ $\mathbf{Z}_{0+}$. If the pair $\left(a_{i}, a_{i+1}\right) \in A_{i} \times A_{i+1}$ satisfies $d\left(a_{i+1}, T a_{i}\right)=D_{i}$ for any $i \in \bar{p}$, then $a_{i+1} \in A_{i+1}$ and $T a_{i} \in B_{i}$ are best proximity points in $A_{i+1}$ and $B_{i}$ with respect to $T: \bigcup_{i \in \bar{p}} A_{i} \rightarrow \bigcup_{i \in \bar{p}} B_{i}$.

In the following, the fact that the best proximity points are best proximity points with respect to the mapping is not mentioned explicitly.

$A_{0 i} \subseteq A_{i}$ is the set of best proximity points of $A_{i}$ and $B_{0 i} \subseteq B_{i}$ is the set of best proximity points of $B_{i}$. Through the paper, it is assumed that $A_{0 i} \neq \varnothing$ and $B_{0 i} \neq \varnothing$, for all $i \in \bar{p}$. An important remark is that the above statement can be considered for the particular case that $B_{i} \equiv A_{i+1}$ which is well known in the context of $p$-cyclic self-mappings $T$ : $\bigcup_{i \in \bar{p}} A_{i} \rightarrow \bigcup_{i \in \bar{p}} B_{i}$ with $T\left(A_{i}\right) \subseteq B_{i}$ for all $i \in \bar{p}$. However, the proposed statement is more general in the sense of the following illustrative example.

Example 1. Consider a metric space $(X, d)$ and $T: A_{1} \cup A_{2} \rightarrow$ $B_{1} \cup B_{2}$ such that $A_{i}, B_{i} \subset X$ are nonempty with $T\left(A_{0 i}\right) \subseteq B_{0 i}$, $D_{i}=d\left(A_{i+1}, B_{i}\right)=d\left(A_{0, i+1}, B_{0 i}\right), D_{A}=d\left(A_{i}, A_{i+1}\right)=$ $d\left(A_{0 i}, A_{0, i+1}\right)$, and $D_{B}=d\left(B_{i}, B_{i+1}\right)=d\left(B_{0 i}, B_{0, i+1}\right)$ for $i=1,2$. Assume also that $A_{01}=\left\{x_{1}, x_{3}\right\} \subset A_{1}, B_{01}=$ $\left\{T x_{1}, T x_{3}\right\} \subset B_{1}, A_{02}=\left\{x_{2}\right\} \subset A_{2}$, and $B_{02}=\left\{T x_{2}\right\} \subset$ $B_{2}$. Then, we can formulate the following simple 2-cyclic proximal-type problem. Fix $x_{1} \in A_{01}$ as a best proximity point of $A_{1}$ and then compute $x_{2} \in A_{02}$ and $x_{3} \in A_{01}$, best proximity points of $A_{2}$ and $A_{1}$, such that

$d\left(x_{2}, T x_{1}\right)=d\left(A_{2}, B_{1}\right)=d\left(A_{02}, B_{01}\right)=D_{1}$ (2-cyclic proximal constraint, first step);

$d\left(x_{3}, T x_{2}\right)=d\left(A_{1}, B_{2}\right)=d\left(A_{01}, B_{02}\right)=D_{2}$ (2-cyclic proximal constraint, second step);

$d\left(A_{1}, A_{2}\right)=d\left(A_{01}, A_{02}\right)=d\left(x_{1}, x_{2}\right)=d\left(x_{2}, x_{3}\right)=$ $D_{A}$ (2-cyclic best proximity constraints);
$d\left(T x_{2}, T x_{1}\right)=d\left(T x_{3}, T x_{2}\right)=D_{B}=d\left(B_{1}, B_{2}\right)=$ $d\left(B_{01}, B_{02}\right)$ (2-cyclic associate best proximity constraints for the images).

Note that there are four potentially distinct constraints related to $D_{1}, D_{2}, D_{A}$, and $D_{B}$ which can be distinct so that the problem is more general than the simple use of $D=$ $d\left(A_{i}, A_{i+1}\right)$ for $i=1,2$ for the 2-cyclic self-mapping $T$ : $A_{1} \cup A_{2} \rightarrow A_{1} \cup A_{2}$. A variant proximal-type problem arises if $A_{1}=B_{2}$ and $A_{2}=B_{1}$ and the best proximity points are taken as follows:

$x_{1} \in A_{01}, T x_{1} \in A_{02}, x_{2} \in A_{01}, T x_{2} \in A_{02}, x_{3} \in A_{01}$, and then $d\left(x_{2}, T x_{1}\right)=d\left(x_{3}, T x_{2}\right)=D_{A}=d\left(A_{1}, A_{2}\right)$.

The following definitions will be then used through the paper.

Definition 2. $T: \bigcup_{i \in \bar{p}} A_{i} \rightarrow \bigcup_{i \in \bar{p}} B_{i}$ is said to be a $p$-cyclic proximal contraction with respect to its domain $\left(\mathrm{CPD}_{p}\right)$ if there are real constants $\alpha_{i} \in[0,1)$, for all $i \in \bar{p}$, such that any two sequences $\left\{x_{n p+i}\right\} \subseteq A_{i+j}$ and $\left\{\bar{x}_{n p+i}\right\} \subseteq A_{i+j}$, for all $i \in \bar{p}$, satisfy the constraints

$$
\begin{array}{r}
d\left(x_{n p+i+1}, \bar{x}_{n p+i}\right) \leq \alpha_{i} d\left(x_{n p+i}, \bar{x}_{n p+i-1}\right)+\left(1-\alpha_{i}\right) D_{A} \\
\forall i \in \bar{p}, \quad \forall n \in \mathbf{Z}_{0+}, \\
d\left(x_{n p+i+1}, \bar{x}_{n p+i+1}\right) \leq \alpha_{i} d\left(x_{n p+i}, \bar{x}_{n p+i}\right), \\
\forall i \in \bar{p}, \quad \forall n \in \mathbf{Z}_{0+},
\end{array}
$$

provided that $x_{0}, \bar{x}_{0} \in A_{j}$, for any given $j \in \bar{p}$ with $A_{i+j}=A_{i+j-p}$ if $i>p-j$ and that $d\left(x_{n p+i+1}, T x_{n p+i}\right)=$ $d\left(\bar{x}_{n p+i+1}, T \bar{x}_{n p+i}\right)=d\left(A_{i+1}, B_{i}\right)=D_{i}$, for all $i \in \bar{p}$.

Definition 3. $T: \bigcup_{i \in \bar{p}} A_{i} \rightarrow \bigcup_{i \in \bar{p}} B_{i}$ is said to be a weak $p$-cyclic proximal contraction with respect to its domain $\left(\mathrm{WCPD}_{p}\right)$ if there are $p$ real constants $\alpha_{i} \geq 0$, for all $i \in \bar{p}$, subject to $\alpha=\prod_{i=1}^{p}\left[\alpha_{i}\right] \in[0,1)$, such that any two sequences $\left\{x_{n p+i}\right\} \subseteq A_{i+j}$ and $\left\{\bar{x}_{n p+i}\right\} \subseteq A_{i+j}$, for all $i \in \bar{p}$, satisfy the constraints (1) and (2) provided that $x_{0}, \bar{x}_{0} \in A_{j}$ for any given $j \in \bar{p}$ and that $d\left(x_{n p+i+1}, T x_{n p+i}\right)=d\left(\bar{x}_{n p+i+1}, T \bar{x}_{n p+i}\right)=D_{i}$, for all $i \in \bar{p}$.

Definition 4. $T: \bigcup_{i \in \bar{p}} A_{i} \rightarrow \bigcup_{i \in \bar{p}} B_{i}$ is said to be a generalized $p$-cyclic proximal contraction with respect to its domain $\left(\mathrm{GCPD}_{p}\right)$ if there are $p$ bounded real functions $\alpha_{i}$ : $A_{i} \rightarrow \mathbf{R}_{0+}$, for all $i \in \bar{p}$, such that any sequences $\left\{x_{n p+i}\right\} \subseteq$ $A_{i+j}$ and $\left\{\bar{x}_{n p+i}\right\} \subseteq A_{i+j}$, for all $i \in \bar{p}$, satisfy the constraints (1) and (2) with the replacements $\alpha_{i} \rightarrow \sup _{x \in A_{i}} \alpha_{i}(x)$, for all $i \in \bar{p}$, provided that $x_{0}, \bar{x}_{0} \in A_{j}$ for any given $j \in \bar{p}$ and that $d\left(x_{n p+i+1}, T x_{n p+i}\right)=d\left(\bar{x}_{n p+i+1}, T \bar{x}_{n p+i}\right)=D_{i}$, for all $i \in \bar{p}$.

Definition 5. $T: \bigcup_{i \in \bar{p}} A_{i} \rightarrow \bigcup_{i \in \bar{p}} B_{i}$ is said to be a generalized weak $p$-cyclic proximal contraction with respect to its domain $\left(\mathrm{GWCPD}_{p}\right)$ if there are $p$ bounded real functions $\alpha_{i}: A_{i} \rightarrow \mathbf{R}_{0+}$, for all $i \in \bar{p}$, and a strictly increasing sequence of integers $\left\{n_{k}\right\}$, subject to $n_{0} \leq \bar{N}_{0}<$ 
$+\infty, \limsup _{k \rightarrow \infty}\left(n_{k+1}-n_{k}\right) \leq \bar{N}<+\infty$, and $\bar{\alpha}=$ $\sup _{k \in \mathbf{Z}_{0+}} \alpha\left(n_{k}, n_{k+1}\right) \in[0,1)$, where

$$
\alpha\left(n_{k}, n_{k+1}\right)=\prod_{j=n_{k}}^{n_{k+1}-1} \prod_{i=1}^{p}\left[\sup _{x_{j+i} \in A_{i}} \alpha_{j p+i}\left(x_{j p+i}\right)\right], \quad \forall k \in \mathbf{Z}_{0+}
$$

such that any two sequences $\left\{x_{n p+i}\right\} \subseteq A_{i+j}$ and $\left\{\bar{x}_{n p+i}\right\} \subseteq$ $A_{i+j}$, for all $i \in \bar{p}$, provided that $x_{0}, \bar{x}_{0} \in A_{j}$ for any given $j \in \bar{p}$, satisfy the constraints

$$
\begin{aligned}
& d\left(x_{n_{k+1} p}, \bar{x}_{n_{k+1} p-1}\right) \\
& \quad \leq \alpha\left(n_{k}, n_{k+1}\right) d\left(x_{n_{k} p+1}, \bar{x}_{n_{k} p}\right)+\left(1-\alpha\left(n_{k}, n_{k+1}\right)\right) D_{A}
\end{aligned}
$$$$
\forall k \in \mathbf{Z}_{0+} \text {, }
$$

$$
\begin{aligned}
& d\left(x_{n_{k+1} p+i}, \bar{x}_{n_{k+1} p+i-1}\right) \\
& \leq\left(\prod_{j=1}^{i-1}\left[\sup _{x \in A_{i}} \alpha_{i}(x)\right]\right) d\left(x_{n_{k+1} p}, \bar{x}_{n_{k+1} p-1}\right) \\
& \quad+\sum_{j=1}^{i-1}\left(\prod_{k=j+1}^{i-1}\left[\sup _{x \in A_{k}} \alpha_{k}(x)\right]\right)\left(1-\sup _{x \in A_{j}} \alpha_{j}(x)\right) D_{A},
\end{aligned}
$$$$
\forall k \in \mathbf{Z}_{0+} \text {, }
$$

$$
\begin{aligned}
& d\left(x_{n_{k+1} p+i}, \bar{x}_{n_{k+1} p+i}\right) \\
& \leq\left(\prod_{j=1}^{i-1}\left[\sup _{x \in A_{i}} \alpha_{i}(x)\right]\right) \alpha\left(n_{k}, n_{k+1}\right) d\left(x_{n_{k} p}, \bar{x}_{n_{k} p}\right),
\end{aligned}
$$

$$
\forall k \in \mathbf{Z}_{0+} \text {, }
$$

and the constraints (1) and (2) provided that $x_{0}, \bar{x}_{0} \in$ $A_{j}$ for any given $j \in \bar{p}$ and that $d\left(x_{n p+i+1}, T x_{n p+i}\right)=$ $d\left(\bar{x}_{n p+i+1}, T \bar{x}_{n p+i}\right)=D_{i}$, for all $i \in \bar{p}$.

The following assertions are obvious without proof from Definitions 2-5.

Assertions 1. If $T: \bigcup_{i \in \bar{p}} A_{i} \rightarrow \bigcup_{i \in \bar{p}} B_{i}$ is $\mathrm{CPD}_{p}$, then it is $\mathrm{WCPD}_{p}$.

If $T: \bigcup_{i \in \bar{p}} A_{i} \rightarrow \bigcup_{i \in \bar{p}} B_{i}$ is $\mathrm{WCPD}_{p}$, then it is $\mathrm{GCPD}_{p}$.

If $T: \bigcup_{i \in \bar{p}} A_{i} \rightarrow \bigcup_{i \in \bar{p}} B_{i}$ is $\mathrm{GCPD}_{p}$, then it is $\mathrm{GWCPD}_{p}$.

Note that the converse implications of those in Assertions 1 are not true in general. The relevant distances satisfy the following convergence and boundedness result.

Lemma 6. Consider a metric space $(X, d)$ with subsets $A_{i}, B_{i} \subset X$ and a $p$-cyclic mapping $T: \bigcup_{i \in \bar{p}} A_{i} \rightarrow \bigcup_{i \in \bar{p}} B_{i}$ which is GWCPD $D_{p}$, subject to $D_{i}=d\left(A_{i+1}, B_{i}\right), D_{A}=$ $d\left(A_{i}, A_{i+1}\right)$, and $D_{B}=d\left(B_{i}, B_{i+1}\right)$, with $T\left(A_{0 i}\right) \subseteq B_{0 i}$, for all $i \in \bar{p}$ such that $A_{0 i} \subseteq A_{i}$ and $B_{0 i} \subseteq B_{i}$ are nonempty, for all $i \in \bar{p}$. Consider also any sequences $\left\{x_{n p+j}\right\},\left\{\bar{x}_{n p+j}\right\} \subseteq \bigcup_{i \in \bar{p}} A_{i}$ which satisfy $d\left(x_{n p+i+1}, T x_{n p+i}\right)=d\left(\bar{x}_{n p+i+1}, T \bar{x}_{n p+i}\right)=D_{i}$, for all $i \in \bar{p}$. Then, the following properties hold.

(i) The sequences of distances $\left\{d\left(x_{n}, \bar{x}_{n+1}\right)\right\} \rightarrow D_{A},\left\{d\left(x_{n}\right.\right.$, $\left.\left.\bar{x}_{n}\right)\right\} \rightarrow 0$ and they are bounded for any given initial points $x_{0}, \bar{x}_{0} \in A_{j} \subset \bigcup_{i \in \bar{p}} A_{i}$, for any given $j \in \bar{p}$.

If, furthermore, $T: \bigcup_{i \in \bar{p}} A_{i} \rightarrow \bigcup_{i \in \bar{p}} B_{i}$ is continuous in $\operatorname{cl} T\left(A_{0 i}\right)$, for all $i \in \bar{p}$, then $\left\{d\left(T x_{n}, T \bar{x}_{n}\right)\right\} \rightarrow 0$ and $\left\{d\left(T x_{n}, T \bar{x}_{n+1}\right)\right\} \rightarrow D_{B}$ and both sequences of distances are bounded for any given initial points $x_{0}, \bar{x}_{0} \in A_{j} \subset \bigcup_{i \in \bar{p}} A_{i}$, for any given $j \in \bar{p}$.

If the sets of best proximity points $A_{0 i}$ and $B_{0 i}$, for all $i \in$ $\bar{p}$, are bounded, then the sequences $\left\{d\left(x_{n}, \bar{x}_{n+1}\right)\right\},\left\{d\left(x_{n}, \bar{x}_{n}\right)\right\}$, $\left\{d\left(T x_{n}, T \bar{x}_{n}\right)\right\}$, and $\left\{d\left(T x_{n}, T \bar{x}_{n+1}\right)\right\}$ are uniformly bounded for any initial points $x_{0}, \bar{x}_{0} \in A_{j} \subset \bigcup_{i \in \bar{p}} A_{i}$ for some $j \in \bar{p}$.

(ii) The sequences $\left\{x_{n p+i}\right\} \subseteq \mathrm{cl} A_{i+j}$, for all $i \in \bar{p}$ (note that $A_{i+j}=A_{i+j-p}$ and $B_{i+j}=B_{i+j-p}$ for $i>p-j$ ) are Cauchy sequences for any initial points any given initial point $x_{0} \in \bigcup_{i \in \bar{p}} A_{i}$ for any arbitrary given $j \in \bar{p}$. The corresponding image sequences $\left\{T x_{n p+i}\right\} \subseteq \mathrm{cl}_{i+j}$, for all $i \in \bar{p}$, are also Cauchy sequences if $T\left(A_{0 i}\right) \subseteq B_{0 i}$, for all $i \in \bar{p}$, and $T$ : $\bigcup_{i \in \bar{p}} A_{i} \rightarrow \bigcup_{i \in \bar{p}} B_{i}$ is continuous in $\operatorname{cl} T\left(A_{0 i}\right)$, for all $i \in \bar{p}$.

Proof. Note that, for any $i \in \bar{p}, \varnothing \neq A_{0 i} \subseteq A_{i}$ implies $A_{i} \neq \varnothing$ and $\varnothing \neq B_{0 i} \subseteq B_{i}$ implies $B_{i} \neq \varnothing$. Take sequences with initial points $x_{0}, \bar{x}_{0} \subset \bigcup_{i \in \bar{p}} A_{i}$ such that $x_{0} \in A_{j}$ and $\bar{x}_{0} \in A_{i}$ for some $i, j \in \bar{p}$. The problem of boundedness and convergence of distances can be described equivalently from initial points $x_{0}, \bar{x}_{0}^{\prime} \in A_{j}$ (i.e., both initial conditions at the same set), with $\bar{x}_{0}^{\prime}=T^{\ell} x_{0}$ and denoting $\bar{x}_{0}^{\prime} \rightarrow \bar{x}_{0}$ for some $\ell \in \overline{p-1} \cup\{0\}$ (in particular, $\ell=0$ if $i=j$ for the set of both initial points) since $\ell \leq p-1$. One has from (1)-(2) and (3)-(6) that, for any sequences $\left\{x_{n p+j+i}\right\} \subseteq A_{j+i}$ and $\left\{\bar{x}_{n p+j+i}\right\} \subseteq A_{j+i}$ fulfilling $\left\{x_{0}\right\} \in A_{j},\left\{\bar{x}_{0}\right\} \in A_{j}$ and $d\left(x_{n p+i+1}, T x_{n p+i}\right)=d\left(\bar{x}_{n p+i+1}, T \bar{x}_{n p+i}\right)=D_{i}$, for all $j, i \in \bar{p}$, such that $A_{j+i}=A_{j+i-p}$ if $i>p-j$ and

$$
\begin{aligned}
D_{A} \leq & d\left(x_{n p+j+1}, \bar{x}_{n p+j}\right) \leq\left(\sup _{x \in A_{j}} \alpha_{j}(x)\right) d\left(x_{n p+j}, \bar{x}_{n p+j-1}\right) \\
& +\left(1-\sup _{x \in A_{j}} \alpha_{j}(x)\right) D_{A}, \quad \forall j \in \bar{p}, \forall n \in \mathbf{Z}_{0+}, \\
D_{A} \leq & d\left(x_{n p+j+2}, \bar{x}_{n p+j+1}\right) \leq\left(\sup _{x \in A_{j+1}} \alpha_{j+1}(x)\right) \\
& \times\left(\left(\sup _{x \in A_{j}} \alpha_{j}(x)\right) d\left(x_{n p+j}, \bar{x}_{n p+j-1}\right)\right. \\
& +\left(1-\sup _{x \in A_{j}} \alpha_{j+1}(x)\right) D_{A}
\end{aligned}
$$




$$
\begin{aligned}
& =D_{A}+\left(\sup _{x \in A_{j+1}} \alpha_{j+1}(x)\right)\left(\sup _{x \in A_{j}} \alpha_{j}(x)\right) \\
& \times\left(d\left(x_{n p+j}, \bar{x}_{n p+j-1}\right)-D_{A}\right), \\
& D_{A} \leq d\left(x_{n p+j+3}, \bar{x}_{n p+j+2}\right) \\
& \leq D_{A}+\left(\sup _{x \in A_{j+2}} \alpha_{j+1}(x)\right) \\
& \times\left(\sup _{x \in A_{j+1}} \alpha_{j+1}(x)\right)\left(\sup _{x \in A_{j}} \alpha_{j}(x)\right) \\
& \times\left(d\left(x_{n p+j}, \bar{x}_{n p+j-1}\right)-D_{A}\right), \\
& D_{A} \leq d\left(x_{n_{k+1} p}, \bar{x}_{n_{k+1} p-1}\right) \leq \alpha\left(n_{k}, n_{k+1}\right) d\left(x_{n_{k} p+1}, \bar{x}_{n_{k} p}\right) \\
& +\left(1-\alpha\left(n_{k}, n_{k+1}\right)\right) D_{A} \\
& \leq D_{A}+\alpha\left(n_{k}, n_{k+1}\right) \alpha\left(n_{k-1}, n_{k-2}\right) \\
& \times\left(d\left(x_{n_{k-1} p+1}, \bar{x}_{n_{k-1} p}\right)-D_{A}\right) \\
& \leq D_{A}+\left(\prod_{j=0}^{k}\left[\alpha\left(n_{j}, n_{j+1}\right)\right]\right)\left(d\left(x_{n_{0} p+1}, \bar{x}_{n_{0} p}\right)-D_{A}\right) \\
& \leq D_{A}+\bar{\alpha}^{k+1}\left(d\left(x_{n_{0} p+1}, \bar{x}_{n_{0} p}\right)-D_{A}\right) \\
& <d\left(x_{n_{0} p+1}, \bar{x}_{n_{0} p}\right)<+\infty, \quad \forall k \in \mathbf{Z}_{0+}, \\
& D_{A} \leq d\left(x_{n_{k+1} p+i}, \bar{x}_{n_{k+1} p+i-1}\right) \\
& \leq D_{A}+\left(\prod_{j=1}^{i-1}\left[\sup _{x \in \bigcup_{j \in \bar{p}} A_{j}} \alpha_{j}(x)\right]\right) \\
& \times\left(\prod_{j=0}^{k}\left[\alpha\left(n_{j}, n_{j+1}\right)\right]\right)\left(d\left(x_{n_{0} p+1}, \bar{x}_{n_{0} p}\right)-D_{A}\right) \\
& \leq D_{A}+\left(\prod_{j=1}^{i-1}\left[\sup _{x \in \bigcup_{j \in \bar{p}} A_{j}} \alpha_{j}(x)\right]\right) \\
& \times \bar{\alpha}^{k+1}\left(d\left(x_{n_{0} p+1}, \bar{x}_{n_{0} p}\right)-D_{A}\right) \\
& \leq D_{A}+\bar{\alpha}^{k+1}\left(1+\bar{N}+\bar{N}^{\prime}\right) \widehat{\alpha}_{M}\left(d\left(x_{n_{0} p+1}, \bar{x}_{n_{0} p}\right)-D_{A}\right)
\end{aligned}
$$

$$
\begin{aligned}
< & D_{A}+\left(\prod_{j=1}^{i-1}\left[\sup _{x \in \bigcup_{j \in \bar{p}} A_{j}} \alpha_{j}(x)\right]\right) \\
& \times\left(d\left(x_{n_{0} p+1}, \bar{x}_{n_{0} p}\right)-D_{A}\right) \\
\leq & \max \left(1, \prod_{j=1}^{i-1}\left[\sup _{x \in \bigcup_{j \in \bar{p}} A_{j}} \alpha_{j}(x)\right]\right) d\left(x_{n_{0} p+1}, \bar{x}_{n_{0} p}\right) \\
\leq & \left(1+\bar{N}+\bar{N}^{\prime}\right) \widehat{\alpha}_{M} d\left(x_{n_{0} p+1}, \bar{x}_{n_{0} p}\right), \quad \forall k \in \mathbf{Z}_{0+},
\end{aligned}
$$

for all $i \in\left(1, \max \left(n_{k+1}-n_{k}\right)\right) \cap \mathbf{Z}_{0+}$ since $\bar{\alpha} \in[0,1)$, $\lim \sup _{k \rightarrow \infty}\left(n_{k+1}-n_{k}\right) \leq \bar{N}<+\infty$ and $\alpha_{i} \in B\left(A_{i} ; \mathbf{R}_{0+}\right)$ with $\max _{i \in \bar{p}}\left(\sup _{x \in A_{i}} \alpha_{i}(x)\right) \leq \widehat{\alpha}_{M}<+\infty$.

Thus, $\exists \lim _{k \rightarrow \infty} d\left(x_{n_{k+1} p+i}, \bar{x}_{n_{k+1} p+i-1}\right)=\lim _{k \rightarrow \infty} d\left(x_{n_{k} p+i}\right.$, $\left.\bar{x}_{n_{k+1} p+i-1}\right)=\lim _{k \rightarrow \infty} d\left(x_{n_{k+1} p+i}, \bar{x}_{n_{k} p+i-1}\right)=D_{A}$, for all

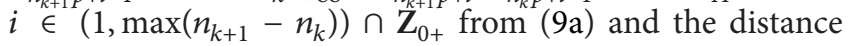
subsequence $\left\{d\left(x_{n_{k+1} p+i}, \bar{x}_{n_{k+1} p+i-1}\right)\right\}$, for all $i \in\left(1, \max \left(n_{k+1}-\right.\right.$ $\left.\left.n_{k}\right)\right) \cap \mathbf{Z}_{0+}$ is bounded from (9b) for any given initial points $x_{0}, \bar{x}_{0} \in \bigcup_{i \in \bar{p}} A_{i}$. Also, one gets from (6), subject to (3), that

$$
\begin{aligned}
& \exists \lim _{k \rightarrow \infty} d\left(x_{n_{k+1} p+i}, \bar{x}_{n_{k+1} p+i}\right) \\
& =\lim _{k \rightarrow \infty} d\left(x_{n_{k} p+i}, \bar{x}_{n_{k+1} p+i}\right)=\lim _{k \rightarrow \infty} d\left(\bar{x}_{n_{k} p+i}, x_{n_{k+1} p+i}\right)=0,
\end{aligned}
$$

for all $i \in\left(1, \max \left(n_{k+1}-n_{k}\right)\right) \cap \mathbf{Z}_{0+}$. Those results also imply that the sequences of distances $\left\{d\left(x_{n}, \bar{x}_{n+1}\right)\right\} \rightarrow$ $D_{A},\left\{d\left(x_{n}, \bar{x}_{n}\right)\right\} \rightarrow 0$. It is now proved by contradiction that $\left\{d\left(T x_{n}, T \bar{x}_{n+1}\right)\right\} \rightarrow D_{B}$ and $\left\{d\left(T x_{n}, T \bar{x}_{n}\right)\right\} \rightarrow 0$. Assume that, for each given $x_{0} \in A_{0 j} \subset \bigcup_{i \in \bar{p}} A_{i}$ for some $j \in \bar{p}$ and any $\varepsilon \in \mathbf{R}_{+}$, there are some $\delta \in \mathbf{R}_{+}$, some $i \in \bar{p}$, some $k_{0}=k_{0}\left(\varepsilon, x_{0}\right) \in \mathbf{Z}_{0+}$, sequences of integers $\left\{n_{k_{\ell}}\right\} \subseteq\left\{n_{k}\right\} \subseteq \mathbf{Z}_{0+}$, and sequences of best proximity points $\left\{x_{n_{k} p+i}^{*}\right\} \subseteq \mathrm{cl}\left(A_{0, i+j}\right)$ and $\left\{T x_{n_{k} p+i}^{*}\right\} \subseteq \operatorname{cl}\left(B_{0, i+j}\right)$ for $k>k_{0}$, such that $\left\{d\left(T x_{n}, T \bar{x}_{n}\right)\right\}$ does not converge to zero so that it has some subsequence which does not converge either:

$$
\begin{gathered}
d\left(A_{i}, A_{i+1}\right)=d\left(A_{0 i}, A_{0, i+1}\right)=D_{A}, \\
d\left(T\left(A_{0 i}\right), T\left(A_{0, i+1}\right)\right)=d\left(B_{i}, B_{i+1}\right)=d\left(B_{0 i}, B_{0, i+1}\right)=D_{B}, \\
d i \in \bar{p}, \\
d\left(x_{n_{k} p+i}, x_{n_{k} p+i-1}^{*}\right)<D_{A}+\varepsilon, \\
\left.\quad \forall x_{n_{k_{\ell}} p+i}, T x_{n_{k_{\ell}} p+i-1}^{*}\right) \geq \delta+D_{B}, \\
d\left(x_{n_{k} p+i}, x_{n_{k} p+i}^{*}\right)<\varepsilon, \quad d\left(T x_{n_{k_{\ell}} p+i}, T x_{n_{k_{\ell}} p+i}^{*}\right) \geq \delta,
\end{gathered}
$$$$
\forall k>k_{0}
$$ 


$$
\begin{array}{r}
T x_{n_{k} p+i}^{*} \in \operatorname{cl} B_{0, i+j} \subseteq \operatorname{cl} B_{i+j}, \\
T x_{n p+i-1} \in \operatorname{cl} B_{0, i+j-1} \subseteq \operatorname{cl} B_{i+j-1}, \\
\forall j \in \bar{p}
\end{array}
$$

since $T\left(A_{0 i}\right) \subseteq B_{0 i}$, for all $i \in \bar{p}$. This implies that $\left\{x_{n_{k_{\ell}} p+i}-\right.$ $\left.x_{n_{k \ell} p+i}^{*}\right\} \rightarrow 0$ while $\left\{T x_{n_{k_{\ell}} p+i}-T x_{n_{k \ell} p+i}^{*}\right\}$ does not converge to zero.

Since $\left\{x_{n_{k_{\rho}} p+i}-x_{n_{k \rho} p+i}^{*}\right\} \rightarrow 0,\left\{x_{n_{k} p+i}^{*}\right\} \subseteq \operatorname{cl}\left(A_{0, i+j}\right)$, $\left\{T x_{n_{k} p+i}^{*}\right\} \subseteq \operatorname{cl}\left(T\left(A_{0, i+j}\right)\right) \subseteq \operatorname{cl}\left(B_{0, i+j}\right)$, and $T: \bigcup_{i \in \bar{p}} A_{i} \rightarrow$ $\bigcup_{i \in \bar{p}} B_{i}$ is continuous in $\mathrm{cl}\left(A_{0, i+j}\right)$, for all $j \in \bar{p}$ and any given $j \in \bar{p}$, then $\left\{T x_{n_{k_{\ell}} p+i}-T x_{n_{k \ell} p+i}^{*}\right\} \rightarrow 0$. Also, $\left\{x_{n_{k} p+i-1}^{*}\right\} \subseteq$ $\mathrm{cl}\left(A_{0, i+j-1}\right),\left\{T x_{n_{k} p+i-1}^{*}\right\} \subseteq \mathrm{cl}\left(T\left(A_{0, i+j-1}\right)\right) \subseteq \mathrm{cl}\left(B_{0, i+j-1}\right)$, for all $i \in \bar{p}$ and any given $j \in \bar{p}$; then $\left\{d\left(T x_{n_{k_{\ell}} p+i}, T x_{n_{k_{\ell}} p+i-1}^{*}\right)\right\} \rightarrow$ $D_{B},\left\{d\left(T x_{n_{k_{\ell}} p+i-1}, T x_{n_{k_{\ell}} p+i}^{*}\right)\right\} \rightarrow D_{B}$. Thus, $\left\{d\left(T x_{n}, T \bar{x}_{n}\right)\right\} \rightarrow$ 0 and $\left\{d\left(T x_{n}, T \bar{x}_{n+1}\right)\right\} \rightarrow 0$ if $x_{0}, \bar{x}_{0} \in A_{j} \subset \bigcup_{i \in \bar{p}} A_{i}$, for any given $j \in \bar{p}$.

On the other hand, $\left\{d\left(T x_{n}, T \bar{x}_{n}\right)\right\}$ and $\left\{d\left(T x_{n}, T \bar{x}_{n+1}\right)\right\}$ are bounded, since $\left\{d\left(x_{n}, \bar{x}_{n}\right)\right\}$ is bounded from (6) because $T$ : $\bigcup_{i \in \bar{p}} A_{i} \rightarrow \bigcup_{i \in \bar{p}} B_{i}$ is $\mathrm{GWCPD}_{p}$ and since one has for some positive real constant $M=M\left(d\left(x_{0}, \bar{x}_{0}\right)\right)$ that

$$
\begin{aligned}
& d\left(T x_{n}, T \bar{x}_{n}\right) \leq d\left(T x_{n}, x_{n}\right)+d\left(\bar{x}_{n}, T \bar{x}_{n}\right)+d\left(x_{n}, \bar{x}_{n}\right) \\
& \quad \leq 2 \max _{1 \leq i \leq p} D_{i}+M, \quad \forall n \in \mathbf{Z}_{0+}, \\
& d\left(T x_{n}, T \bar{x}_{n+1}\right) \\
& \quad \leq d\left(T x_{n}, x_{n+1}\right)+d\left(\bar{x}_{n+1}, T \bar{x}_{n+1}\right)+d\left(x_{n+1}, \bar{x}_{n+1}\right) \\
& \quad \leq 2 \max _{1 \leq i \leq p} D_{i}+M, \quad \forall n \in \mathbf{Z}_{0+} .
\end{aligned}
$$

If the sets of best proximity points $A_{0 i}$ for all $i \in \bar{p}$ are bounded, then the sequences of distances $\left\{d\left(x_{n}, \bar{x}_{n+1}\right)\right\}$, $\left\{d\left(x_{n}, \bar{x}_{n}\right)\right\},\left\{d\left(T x_{n}, T \bar{x}_{n}\right)\right\}$, and $\left\{d\left(T x_{n}, T \bar{x}_{n+1}\right)\right\}$ are uniformly bounded for any initial best proximity points $x_{0}, \bar{x}_{0} \in$ $A_{0 j} \subset \bigcup_{i \in \bar{p}} A_{i}$ for some $j \in \bar{p}$ which follows by taking $M=M \sup _{x_{0}, \bar{x}_{0} \in \bigcup_{i \in \bar{p}} A_{i}}\left(d\left(x_{0}, \bar{x}_{0}\right)\right)$. Property (i) has been fully proved.

To prove Property (ii), take any sequences $\left\{x_{n p+i}\right\} \subseteq A_{0, i+j}$ and $\left\{\bar{x}_{n p+i}\right\} \subseteq A_{0, i+j}$, for all $i \in \bar{p}$, for given initial points $x_{0}, \bar{x}_{0} \in A_{j} \subset \bigcup_{i \in \bar{p}} A_{i}$ for some $j \in \bar{p}$. Note, from (6) for $\left\{\bar{x}_{n_{k+1} p+i}\right\}=\left\{x_{n_{k} p+i}\right\}$, for all $i \in \bar{p}$, that

$$
\begin{aligned}
& d\left(x_{n_{k+1} p+i}, x_{n_{k} p+i}\right) \\
& \leq H d\left(x_{n_{k} p}, x_{n_{k-1} p}\right) \leq h_{0} \bar{\alpha} d\left(x_{n_{k} p}, x_{n_{k-1} p}\right), \\
& \quad \forall k \in \mathbf{Z}_{0+}, \quad \forall i \in \bar{p},
\end{aligned}
$$

$$
\begin{aligned}
& d\left(x_{n_{k+1} p+i+\ell h}, x_{n_{k} p+i}\right) \\
& \quad \leq h_{0} \bar{\alpha}\left(1+\bar{\alpha}+\cdots+\bar{\alpha}^{\ell}\right) d\left(x_{n_{k} p}, x_{n_{k-1} p}\right) \\
& d\left(x_{n_{k+1} p+i+\ell h}, x_{n_{k} p+i}\right) \\
& \quad \leq h_{0} \frac{\bar{\alpha}\left(1-\bar{\alpha}^{\ell+1}\right)}{1-\bar{\alpha}} d\left(x_{n_{k} p}, x_{n_{k-1} p}\right) \\
& \quad \leq h_{0} \frac{\bar{\alpha}}{1-\bar{\alpha}} d\left(x_{n_{k} p}, x_{n_{k-1} p}\right), \quad \forall k, \ell \in \mathbf{Z}_{0+}, \forall i \in \bar{p},
\end{aligned}
$$

with the given upper bound being independent of the integers $\ell$ and $i$. Thus, one has for any $\mathbf{Z}_{0+}$ that

$$
\begin{array}{r}
d\left(x_{n_{k+m+1} p+i+\ell j}, x_{n_{k+m} p+i}\right) \leq \bar{\alpha}^{m} h_{0} \frac{\bar{\alpha}}{1-\bar{\alpha}} d\left(x_{n_{k} p}, x_{n_{k-1} p}\right) \\
\forall k \in \mathbf{Z}_{0+}, \quad \forall i \in \bar{p}, \quad \forall j(\leq h), \ell \in \mathbf{Z}_{0+}
\end{array}
$$

where $H=\sup _{k \in \mathbf{Z}_{0+}}\left(\prod_{j=1}^{p}\left[\sup _{x \in A_{i}} \alpha_{i}(x)\right] \alpha\left(n_{k}, n_{k+1}\right)\right) \leq h_{0} \bar{\alpha}$, with $h_{0} \geq \sup _{k \in \mathbf{Z}_{0+}}\left(\prod_{j=1}^{p}\left[\sup _{x \in A_{i}} \alpha_{i}(x)\right]\right)$ and $1 \leq j \leq h=$ $\max \left(1, \sup _{k \in \mathbf{Z}_{0+}}\left(n_{k+1}-n_{k}\right)\right)$, since Definition 5 holds for $\bar{\alpha}=$ $\sup _{k \in \mathbf{Z}_{0+}} \alpha\left(n_{k}, n_{k+1}\right) \in[0,1)$. Thus, one gets from (15) that, for any given real $\varepsilon \in \mathbf{R}_{+}, d\left(x_{n_{k+m+1} p+i+\ell j}, x_{n_{k+m} p+i}\right)<\varepsilon$, for all $k, \ell, j(\leq h) \in \mathbf{Z}_{0+}$ for any $i \in \bar{p}$ and any given integers $n_{k-1}, n_{k} \in \mathbf{Z}_{0+}$ if $m\left(\in \mathbf{Z}_{0+}\right)>\ln \left(h_{0} d\left(x_{n_{k} p}, x_{n_{k-1} p}\right) /(1-\right.$ $\bar{\alpha}) \varepsilon) /|\ln \alpha|-1$. Thus, the sequences $\left\{x_{n p+i}\right\} \subseteq \operatorname{cl} A_{0 i}$, for all $i \in \bar{p}$, are Cauchy sequences for any given initial point $x_{0} \in A_{0 j} \subset \bigcup_{i \in \bar{p}} A_{i}$ and any $j \in \bar{p}$. This implies also that the sequences of images of the above points are also Cauchy sequences since $T: \bigcup_{i \in \bar{p}} A_{i} \rightarrow \bigcup_{i \in \bar{p}} B_{i}$ is contractive and then continuous.

From Assertions 1, we also have the subsequent parallel result to Lemma 6.

Lemma 7. Assume that $T: \bigcup_{i \in \bar{p}} A_{i} \rightarrow \bigcup_{i \in \bar{p}} B_{i}$ is either $G C P D_{p}$ or $W C P D_{p}$ or $C P D_{p}$ with the assumptions of Lemma 6, and consider any sequences $\left\{x_{n p+j}\right\}$, $\left\{\bar{x}_{n p+j}\right\} \subseteq \bigcup_{i \in \bar{p}} A_{i}$ which satisfy $d\left(x_{n p+i+1}, T x_{n p+i}\right)=$ $d\left(\bar{x}_{n p+i+1}, T \bar{x}_{n p+i}\right)=D_{i}$, for all $i \in \bar{p}$. Then, the following properties hold.

(i) The sequences of distances $\left\{d\left(x_{n}, \bar{x}_{n+1}\right)\right\} \rightarrow D_{A}$, $\left\{d\left(x_{n}, \bar{x}_{n}\right)\right\} \rightarrow 0$ and they are bounded for any given initial points $x_{0}, \bar{x}_{0} \in A_{j} \subset \bigcup_{i \in \bar{p}} A_{i}$ for any given $j \in \bar{p}$.

If, furthermore, $T\left(A_{0 i}\right) \subseteq B_{0 i}$, for all $i \in \bar{p}$, and $T$ : $\bigcup_{i \in \bar{p}} A_{i} \rightarrow \bigcup_{i \in \bar{p}} B_{i}$ is continuous in $\mathrm{cl} T\left(A_{0 i}\right)$, for all $i \in \bar{p}$, then $\left\{d\left(T x_{n}, T \bar{x}_{n}\right)\right\} \rightarrow 0$ and $\left\{d\left(T x_{n}, T \bar{x}_{n+1}\right)\right\} \rightarrow D_{B}$ and it is bounded for any initial points for any given initial points $x_{0}, \bar{x}_{0} \in A_{0 j} \subset \bigcup_{i \in \bar{p}} A_{i}$, for some $j \in \bar{p}$.

If the sets of best proximity points $A_{0 i}$ and $B_{0 i}$, for all $i \epsilon$ $\bar{p}$, are bounded, then the sequences $\left\{d\left(x_{n}, \bar{x}_{n+1}\right)\right\},\left\{d\left(x_{n}, \bar{x}_{n}\right)\right\}$, $\left\{d\left(T x_{n}, T \bar{x}_{n}\right)\right\}$, and $\left\{d\left(T x_{n}, T \bar{x}_{n+1}\right)\right\}$ are uniformly bounded for any initial best proximity points $x_{0}, \bar{x}_{0} \in A_{0 j} \subset \bigcup_{i \in \bar{p}} A_{i}$ for some $j \in \bar{p}$.

(ii) The sequences $\left\{x_{n p+i}\right\} \subseteq \operatorname{cl} A_{0, i+j}$, for all $i \in \bar{p}$, are Cauchy sequences for any given initial point $x_{0} \in A_{0 j} \subset$ 
$\bigcup_{i \in \bar{p}} A_{i}$ for any given $j \in \bar{p}$. The corresponding image sequences $\left\{T x_{n p+i}\right\} \subseteq \mathrm{cl}_{0, i+j}$, for all $i \in \bar{p}$, are also convergent, then Cauchy sequences if $T\left(A_{0 i}\right) \subseteq B_{0 i}$, for all $i \in \bar{p}$, and $T$ : $\bigcup_{i \in \bar{p}} A_{i} \rightarrow \bigcup_{i \in \bar{p}} B_{i}$ is continuous in $\mathrm{cl} T\left(A_{0 i}\right)$, for all $i \in \bar{p}$.

Definition 8. T: $\bigcup_{i \in \bar{p}} A_{i} \rightarrow \bigcup_{i \in \bar{p}} B_{i}$ is said to be a $p$-cyclic proximal contraction with respect to its image $\left(\mathrm{CPI}_{p}\right)$ if there are real constants $\beta_{i} \in[0,1)$, for all $i \in \bar{p}$, such that any two sequences $\left\{T x_{n p+i}\right\} \subseteq T\left(A_{i+j}\right) \subseteq B_{i+j}$ and $\left\{\bar{x}_{n p+i}\right\} \subseteq T\left(A_{i+j}\right) \subseteq$ $B_{i+j}$, for all $i \in \bar{p}$, being point-to-point images of sequences $\left\{x_{n p+i}\right\} \subseteq A_{i+j}$ and $\left\{\bar{x}_{n p+i}\right\} \subseteq A_{i+j}$ for any given $j \in \bar{p}$ which satisfy $d\left(x_{n p+i+1}, T x_{n p+i}\right)=d\left(\bar{x}_{n p+i+1}, T \bar{x}_{n p+i}\right)=D_{i}$, for all $i \in$ $\bar{p}$, where $B_{i+j}=B_{i+j-p}$ if $i>p-j$ such that the initial points $T x_{0}, T \bar{x}_{0} \in T\left(A_{j}\right) \subseteq B_{j}$ are the images of points $x_{0}, \bar{x}_{0} \in A_{j}$, for any given $j \in \bar{p}$, satisfy the constraints

$$
\begin{array}{r}
d\left(T x_{n p+i+1}, T \bar{x}_{n p+i}\right) \\
\leq \beta_{i} d\left(T x_{n p+i}, T \bar{x}_{n p+i-1}\right)+\left(1-\beta_{i}\right) D_{B}, \\
\forall i \in \bar{p}, \quad \forall n \in \mathbf{Z}_{0+}, \\
d\left(T x_{n p+i+1}, T \bar{x}_{n p+i+1}\right) \leq \beta_{i} d\left(T x_{n p+i}, T \bar{x}_{n p+i}\right), \\
\forall i \in \bar{p}, \quad \forall n \in \mathbf{Z}_{0+} .
\end{array}
$$

Definition 9. $T: \bigcup_{i \in \bar{p}} A_{i} \rightarrow \bigcup_{i \in \bar{p}} B_{i}$ is said to be a weak $p$-cyclic proximal contraction with respect to its image $\left(\mathrm{WCPI}_{p}\right)$ if there are $p$ real constants $\beta_{i} \geq 0$, for all $i \in \bar{p}$, subject to $\beta=\prod_{i=1}^{p}\left[\beta_{i}\right] \in[0,1)$, such that any two sequences $\left\{T x_{n p+i}\right\} \subseteq T\left(A_{i+j}\right) \subseteq B_{i+j}$ and $\left\{T \bar{x}_{n p+i}\right\} \subseteq T\left(A_{i+j}\right) \subseteq B_{i+j}$, for all $i \in \bar{p}$, being point-to-point images of sequences $\left\{x_{n p+i}\right\} \subseteq$ $A_{i+j}$ and $\left\{\bar{x}_{n p+i}\right\} \subseteq A_{i+j}$ for any given $j \in \bar{p}$, where $B_{i+j}=$ $B_{i+j-p}$ for $i>p-j$, for all $i \in \bar{p}$, such that the initial points $T x_{0}, T \bar{x}_{0} \in T\left(A_{j}\right) \subseteq B_{j}$ are the images of points $x_{0}, \bar{x}_{0} \in A_{j}$, for any given $j \in \bar{p}$, satisfy constraints (16) and (17).

Definition 10. $T: \bigcup_{i \in \bar{p}} A_{i} \rightarrow \bigcup_{i \in \bar{p}} B_{i}$ is said to be a generalized $p$-cyclic proximal contraction with respect to its image $\left(\mathrm{GCPI}_{p}\right)$ if there are $p$ bounded real functions $\beta_{i}$ : $A_{i} \rightarrow \mathbf{R}_{0+}$, for all $i \in \bar{p}$, such that any sequences $\left\{T x_{n p+i}\right\} \subseteq$ $T\left(A_{i+j}\right) \subseteq B_{i+j}$ and $\left\{T \bar{x}_{n p+i}\right\} \subseteq T\left(A_{i+j}\right) \subseteq B_{i+j}$, for all $i \in \bar{p}$, being point-to-point images of sequences $\left\{x_{n p+i}\right\} \subseteq A_{i+j}$ and $\left\{\bar{x}_{n p+i}\right\} \subseteq A_{i+j}$ for any given $j \in \bar{p}$, where $B_{i+j}=B_{i+j-p}$ for $i>p-j$, for all $i \in \bar{p}$, such that the initial points $T x_{0}, T \bar{x}_{0} \in T\left(A_{j}\right) \subseteq B_{j}$ are the images of points $x_{0}, \bar{x}_{0} \in A_{j}$, satisfy the constraints (16) and (17) with the replacements $\beta_{i} \rightarrow \sup _{x \in A_{i}} \beta_{i}(T x)$, for all $i \in \bar{p}$.

Definition 11. $T: \bigcup_{i \in \bar{p}} A_{i} \rightarrow \bigcup_{i \in \bar{p}} B_{i}$ is said to be a generalized weak $p$-cyclic proximal contraction with respect to its image (GWCPI ${ }_{p}$ ) if there are $p$ bounded real functions $\beta_{i}$ : $A_{i} \rightarrow \mathbf{R}_{0+}$, for all $i \in \bar{p}$, and a strictly increasing sequence of integers $\left\{n_{k}\right\}$, subject to $n_{0} \leq \bar{N}_{0}<+\infty, \lim \sup _{k \rightarrow \infty}\left(n_{k+1}-\right.$ $\left.n_{k}\right) \leq \bar{N}<+\infty$, and $\bar{\alpha}=\sup _{k \in \mathbf{Z}_{0+}} \beta\left(n_{k}, n_{k+1}\right) \in[0,1)$, where $\beta\left(n_{k}, n_{k+1}\right)=\prod_{j=n_{k}}^{n_{k+1}-1} \prod_{i=1}^{p}\left[\sup _{x_{j+i} \in A_{i}} \beta_{j p+i}\left(T x_{j p+i}\right)\right], \quad \forall k \in \mathbf{Z}_{0+}$,

such that any two sequences $\left\{T x_{n p+i}\right\} \subseteq T\left(A_{i+j}\right) \subseteq B_{i+j}$ and $\left\{\bar{x}_{n p+i}\right\} \subseteq T\left(A_{i+j}\right) \subseteq B_{i+j}$, for all $i \in \bar{p}$, being point-to-point images of sequences $\left\{x_{n p+i}\right\} \subseteq A_{i+j}$ and $\left\{\bar{x}_{n p+i}\right\} \subseteq A_{i+j}$ for any given $j \in \bar{p}$, where $B_{i+j}=B_{i+j-p}$ for $i>p-j$, for all $i \in \bar{p}$, such that the initial points $T x_{0}, T \bar{x}_{0} \in T\left(A_{j}\right) \subseteq B_{j}$ are the images of points $x_{0}, \bar{x}_{0} \in A_{j}$, for any given $j \in \bar{p}$, satisfy the following constraints:

$$
\begin{aligned}
& d\left(T x_{n_{k+1} p}, T \bar{x}_{n_{k+1} p-1}\right) \\
& \quad \leq \beta\left(n_{k}, n_{k+1}\right) d\left(T x_{n_{k} p+1}, T \bar{x}_{n_{k} p}\right)+\left(1-\beta\left(n_{k}, n_{k+1}\right)\right) D_{B}
\end{aligned}
$$

$$
\begin{aligned}
& d\left(T x_{n_{k+1} p+i}, T \bar{x}_{n_{k+1} p+i-1}\right) \\
& \leq\left(\prod_{j=1}^{i-1}\left[\sup _{x \in A_{i}} \beta_{i}(T x)\right]\right) d\left(T x_{n_{k+1} p}, T \bar{x}_{n_{k+1} p-1}\right) \\
& \quad+\sum_{j=1}^{i-1}\left(\prod_{k=j+1}^{i-1}\left[\sup _{x \in A_{k}} \beta_{k}(T x)\right]\right)\left(1-\sup _{x \in A_{j}} \beta_{j}(x)\right) D_{B},
\end{aligned}
$$

$\forall k \in \mathbf{Z}_{0+}$,

$$
\begin{aligned}
& d\left(T x_{n_{k+1} p+i}, T \bar{x}_{n_{k+1} p+i}\right) \\
& \quad \leq\left(\prod_{j=1}^{i-1}\left[\sup _{x \in A_{i}} \beta_{i}(T x)\right]\right) \beta\left(n_{k}, n_{k+1}\right) d\left(T x_{n_{k} p}, T \bar{x}_{n_{k} p}\right),
\end{aligned}
$$

$\forall k \in \mathbf{Z}_{0+}$,

and constraints (16) and (17) with the replacements $\beta_{i} \rightarrow$ $\sup _{x \in A_{i}} \beta_{i}(T x)$, for all $i \in \bar{p}$.

The following assertions are obvious without proof from Definitions 8-11 and are a parallel result to Assertions 1.

Assertions 2. If $T: \bigcup_{i \in \bar{p}} A_{i} \rightarrow \bigcup_{i \in \bar{p}} B_{i}$ is $\mathrm{CPI}_{p}$, then it is WCPI $_{p}$.

If $T: \bigcup_{i \in \bar{p}} A_{i} \rightarrow \bigcup_{i \in \bar{p}} B_{i}$ is $\mathrm{WCPI}_{p}$, then it is $\mathrm{GCPI}_{p}$.

If $T: \bigcup_{i \in \bar{p}} A_{i} \rightarrow \bigcup_{i \in \bar{p}} B_{i}$ is $\mathrm{GCPI}_{p}$, then it is $\mathrm{GWCPI}_{p}$.

Note that the converse implications of those in Assertions 1 are not true in general.

The relevant distances satisfy the following convergence and boundedness result which is a counterpart of Lemmas 6 and 7. Its proof is close to that of Lemma 6 and Assertions 2 by using (16) and (17) for Definition 11 and their variants for Definitions 8-10. 
Lemma 12. Assume that $T: \bigcup_{i \in \bar{p}} A_{i} \rightarrow \bigcup_{i \in \bar{p}} B_{i}$ is either $G W C P I_{p}$ or $G C P I_{p}$ or $W C P I_{p}$ or $C P I_{p}$ under the assumptions of Lemma 6 , and consider any sequences $\left\{x_{n p+j}\right\},\left\{\bar{x}_{n p+j}\right\} \subseteq \bigcup_{i \in \bar{p}} A_{i}$ which satisfy $d\left(x_{n p+i+1}, T x_{n p+i}\right)=$ $d\left(\bar{x}_{n p+i+1}, T \bar{x}_{n p+i}\right)=D_{i}$, for all $i \in \bar{p}$. Then, the following properties hold.

(i) The sequences of distances $\left\{d\left(x_{n}, \bar{x}_{n+1}\right)\right\} \rightarrow D_{A}$, $\left\{d\left(x_{n}, \bar{x}_{n}\right)\right\} \rightarrow 0$ and they are bounded for any given initial points $x_{0}, \bar{x}_{0} \in A_{j} \subset \bigcup_{i \in \bar{p}} A_{i}$, for some $j \in \bar{p}$.

If, furthermore, $T\left(A_{0 i}\right) \subseteq B_{0 i}$, for all $i \in \bar{p}$, and $T$ : $\bigcup_{i \in \bar{p}} A_{i} \rightarrow \bigcup_{i \in \bar{p}} B_{i}$ is continuous in $\operatorname{cl} T\left(A_{0 i}\right)$, for all $i \in \bar{p}$, then $\left\{d\left(T x_{n}, T \bar{x}_{n}\right)\right\} \rightarrow 0$ and $\left\{d\left(T x_{n}, T \bar{x}_{n+1}\right)\right\} \rightarrow D_{B}$ and it is bounded any given initial points $x_{0}, \bar{x}_{0} \in A_{0 j} \subset \bigcup_{i \in \bar{p}} A_{i}$ for some $j \in \bar{p}$.

If the sets of best proximity points $A_{0 i}$ and $B_{0 i}$, for all $i \in$ $\bar{p}$, are bounded, then the sequences $\left\{d\left(x_{n}, \bar{x}_{n+1}\right)\right\},\left\{d\left(x_{n}, \bar{x}_{n}\right)\right\}$, $\left\{d\left(T x_{n}, T \bar{x}_{n}\right)\right\}$, and $\left\{d\left(T x_{n}, T \bar{x}_{n+1}\right)\right\}$ are uniformly bounded for any initial best proximity points $x_{0}, \bar{x}_{0} \in A_{0 j} \subset \bigcup_{i \in \bar{p}} A_{i}$ for some $j \in \bar{p}$.

(ii) The sequences $\left\{x_{n p+i}\right\} \subseteq \mathrm{cl}_{0, i+j}$, for all $i \in \bar{p}$, are Cauchy sequences for any given initial point $x_{0} \in A_{0 j} \subset$ $\bigcup_{i \in \bar{p}} A_{i}$ for any given $j \in \bar{p}$. The corresponding image sequences $\left\{T x_{n p+i}\right\} \subseteq \mathrm{cl} B_{0, i+j}$, for all $i \in \bar{p}$, are also convergent; then Cauchy sequences if $T\left(A_{0 i}\right) \subseteq B_{0 i}$, for all $i \in \bar{p}$, and $T: \bigcup_{i \in \bar{p}} A_{i} \rightarrow \bigcup_{i \in \bar{p}} B_{i}$ are continuous in $\operatorname{cl} T\left(A_{0 i}\right)$, for all $i \in \bar{p}$.

Remark 13. The result $\left\{d\left(T x_{n}, T \bar{x}_{n+1}\right)\right\} \rightarrow D_{B}$ of Lemma 12, as well as Lemma 12(ii), obtained under the assumption that $T: \bigcup_{i \in \bar{p}} A_{i} \rightarrow \bigcup_{i \in \bar{p}} B_{i}$ is continuous in $\operatorname{cl} T\left(A_{0 i}\right)$ and also holds without such a continuity assumption if the contractive conditions (16) and (17) become modified to the right limits as follows:

$$
\begin{array}{r}
d\left(T x_{n p+i+1}^{+}, T \bar{x}_{n p+i}^{+}\right) \\
\leq \beta_{i} d\left(T x_{n p+i}^{+}, T \bar{x}_{n p+i-1}^{+}\right)+\left(1-\beta_{i}\right) D_{B}, \\
\forall i \in \bar{p}, \quad \forall n \in \mathbf{Z}_{0+}, \\
d\left(T x_{n p+i+1}^{+}, T \bar{x}_{n p+i+1}^{+}\right) \leq \beta_{i} d\left(T x_{n p+i}^{+}, T \bar{x}_{n p+i}^{+}\right), \\
\forall i \in \bar{p}, \quad \forall n \in \mathbf{Z}_{0+},
\end{array}
$$

provided that any discontinuity points in $\bigcup_{i \in p} \operatorname{clT}\left(A_{0 i}\right)$, if any, are of first-class finite-jump type under right best proximity constraints

$$
d\left(x_{n p+i+1}, T x_{n p+i}^{+}\right)=d\left(\bar{x}_{n p+i+1}, T \bar{x}_{n p+i}^{+}\right)=D_{i}, \quad \forall i \in \bar{p} .
$$

In the same way, the result $\left\{d\left(T x_{n}, T \bar{x}_{n+1}\right)\right\} \rightarrow D_{B}$ of Lemmas 6 and 7 , as well as their properties (ii) obtained under the assumption that $T: \bigcup_{i \in \bar{p}} A_{i} \rightarrow \bigcup_{i \in \bar{p}} B_{i}$, is continuous in $\operatorname{cl} T\left(A_{0 i}\right)$ and also holds under finite-jump discontinuities in $\operatorname{cl} T\left(A_{0 i}\right)$ for sequences $\left\{x_{n}\right\},\left\{\bar{x}_{n}\right\},\left\{T x_{n}\right\}$, and $\left\{T \bar{x}_{n}\right\}$ satisfying the contractive proximal conditions (1) and (2) if Definition 2, or their counterparts of Definitions 3-5 for right values $T x_{n}^{+}$ and $T \bar{x}_{n}^{+}$under right best proximity constraints (24).

\section{Best Proximity Points and Related Convergence Results}

We first recall the subsequent useful definition $[2-4,7]$ as follows.

Definition 14. Let $A$ and $B$ be two nonempty subsets of a metric space $(X, d)$ and let $d(y, A)=\inf \{d(y, x): x \in A\}$ for $y \in X . A$ is said to be approximately compact with respect to $B$ if each sequence $\left\{x_{n}\right\} \subset A$ satisfying $\left\{d\left(y, x_{n}\right)\right\} \rightarrow d(y, A)$ for some $y \in B$ has a convergent subsequence.

Note that if the sets of best proximity points $A_{0} \subseteq A$ and $B_{0} \subseteq B$ are nonempty if Definition 14 holds, then $A$ is approximately compact with respect to $B$ if every sequence $\left\{x_{n}\right\} \subset A$ such that $\left\{d\left(y, x_{n}\right)\right\} \rightarrow D$ for some $y \in B_{0}$ has a convergent subsequence $\left\{x_{n_{k}}\right\} \subseteq\left\{x_{n}\right\}$ since $D=$ $d(y, A)=d\left(B_{0}, A\right)=d\left(B_{0}, A_{0}\right)$. Note that every set is approximately compact with respect to itself and that every compact set is approximately compact with respect to any nonempty subset of a metric space. Also, if $B$ is compact and $A$ is approximately compact with respect to $B$, each sequence $\left\{x_{n}\right\} \subset A$ has a convergent sequence. If $A$ and $B$ are nonempty and closed and $A$ is approximately compact with respect to $B$, then $B_{0}$ is closed. See, for instance, $[2-4,7]$. A result on existence and uniqueness of best proximity points follows for $p$-cyclic proximal contraction fulfilling Definitions $2-5$ under Lemmas 6 and 7 follows.

Theorem 15. Consider a complete metric space $(X, d)$ with nonempty closed subsets $A_{i}, B_{i} \subset X$ and a p-cyclic mapping $T: \bigcup_{i \in \bar{p}} A_{i} \rightarrow \bigcup_{i \in \bar{p}} B_{i}$ being either $G W C P D_{p}$ or $G C P D_{p}$ or $W C P D_{p}$ or $C P D_{p}$, subject to set distances $D_{i}=d\left(A_{i+1}, B_{i}\right)$, $D_{A}=d\left(A_{i}, A_{i+1}\right)$, and $D_{B}=d\left(B_{i}, B_{i+1}\right)$, for all $i \in \bar{p}$ such that $A_{0 i}$ is nonempty and $B_{i}$ is approximately compact with respect to $A_{i}$ and $T\left(A_{0 i}\right) \subseteq B_{0 i}$, for all $i \in \bar{p}$. The following properties hold.

(i) $T: \bigcup_{i \in \bar{p}} A_{i} \rightarrow \bigcup_{i \in \bar{p}} B_{i}$ has a unique best proximity point $x_{i}^{*}$ at $A_{i}$ such that $d\left(x_{i}^{*}, x_{i+1}^{*}\right)=D_{A}$, for all $i \in$ $\bar{p}$, and all the sequences $\left\{x_{n}\right\} \subset \bigcup_{i \in \bar{p}} A_{i}$ converge to a unique limit cycle $\left\{x_{1}^{*}, x_{2}^{*}, \ldots, x_{p}^{*}\right\}$.

(ii) Furthermore, if $T: \bigcup_{i \in \bar{p}} A_{i} \rightarrow \bigcup_{i \in \bar{p}} B_{i}$ either is continuous, respectively, has eventual finite-jump discontinuity points, then $z_{i}^{*}=T x_{i}^{*}$, for all $i \in \bar{p}$, respectively, $z_{i}^{*^{+}}=\left(T x_{i}^{*}\right)^{+}$, for all $i \in \bar{p}$, are unique best proximity points such that $d\left(T x_{i}^{*}, T x_{i+1}^{*}\right)=D_{B}$, for all $i \in \bar{p}$, respectively, $d\left(T x_{i}^{*^{+}}, T x_{i+1}^{*^{+}}\right)=D_{B}$, for all $i \in \bar{p}$, and all the sequences $\left\{x_{n}\right\} \subset \bigcup_{i \in \bar{p}} A_{i}$ converge to a unique limit cycle $\left\{T x_{1}^{*^{+}}, T x_{2}^{*^{+}}, \ldots, T x_{p}^{*^{+}}\right\}$.

Proof. Since $A_{0 i}$ is nonempty and $T\left(A_{0 i}\right) \subseteq B_{0 i}$, for all $i \in \bar{p}$, then $T\left(A_{0 i}\right)$ and $B_{0 i}$ are nonempty, for all $i \in \bar{p}$. Also, $A_{0 i}$ is closed since $B_{i}$ is approximately compact with respect to 
$A_{i}$. Consider any sequences $\left\{x_{n p+j}\right\},\left\{\bar{x}_{n p+j}\right\} \subseteq \bigcup_{i \in \bar{p}} A_{i}$ which satisfy

$$
d\left(x_{n p+i+1}, T x_{n p+i}\right)=d\left(\bar{x}_{n p+i+1}, T \bar{x}_{n p+i}\right)=D_{i}, \quad \forall i \in \bar{p} .
$$

One gets, from Lemma 6(i), if the mapping $T: \bigcup_{i \in \bar{p}} A_{i} \rightarrow$ $\bigcup_{i \in \bar{p}} B_{i}$ is $\mathrm{GWCPD}_{p}$ and, from Lemma 7(i), if the mapping $T: \bigcup_{i \in \bar{p}} A_{i} \rightarrow \bigcup_{i \in \bar{p}} B_{i}$ is either $\mathrm{GCPD}_{p}$ or $\mathrm{WCPD}_{p}$ or $\mathrm{CPD}_{p}$ that, since $T\left(A_{0 i}\right) \subseteq B_{0 i}$, for all $i \in \bar{p}$,

$$
\begin{gathered}
\left\{d\left(\bar{x}_{p(n+1)+i+j}, x_{p n+i+j}\right)\right\} \longrightarrow 0, \\
\left\{d\left(\bar{x}_{p(n+1)+i+j+1}, x_{p n+i+j+1}\right)\right\} \longrightarrow 0, \\
\left\{d\left(\bar{x}_{p n+i+j}, x_{p n+i+j+1}\right)\right\} \\
\longrightarrow D_{A}=d\left(y_{i+j}, A_{i+1}\right)=d\left(y_{i+j}, A_{0, i+1}\right) \\
=d\left(y_{i+j}, x_{p n_{k}+i+j+1}\right), \\
\left\{d\left(\bar{x}_{p n+i+j+1}, T x_{p n+i+j}\right)\right\} \\
\longrightarrow D_{i}=d\left(y_{i+j+1}, B_{i}\right)=d\left(y_{i+j+1}, T\left(A_{i}\right)\right) \\
=d\left(y_{i+j+1}, T\left(A_{0 i}\right)\right)=d\left(y_{i+j+1}, B_{0 i}\right) \\
=d\left(y_{i+j+1}, T x_{p n_{k}+i+j}\right), \quad \forall i \in \bar{p},
\end{gathered}
$$

for some $y_{i+j} \in A_{0, i+j}$ and $y_{i+j+1} \in A_{0, i+j+1}$ since $D_{A}=$ $d\left(A_{i}, A_{i+1}\right)=d\left(A_{0 i}, A_{0, i+1}\right)$ and $D_{i}=d\left(A_{i+1}, B_{i}\right)=$ $d\left(A_{0 i+1}, B_{0 i}\right)$, for all $i \in \bar{p}$, and some subsequences

$$
\begin{gathered}
\left\{\bar{x}_{p n_{k}+i+j+1}\right\},\left\{x_{p n_{k}+i+j+1}\right\} \subseteq A_{0, i+j+1}, \\
\left\{\bar{x}_{p n_{k}+i+j}\right\},\left\{x_{p n_{k}+i+j}\right\} \subseteq A_{0, i+j}
\end{gathered}
$$

of the sequences $\left\{\bar{x}_{p n+i+j+1}\right\},\left\{x_{p n+i+j+1}\right\} \subseteq A_{i+j+1}$ and $\left\{\bar{x}_{p n+i+j}\right\},\left\{x_{p n+i+j}\right\} \subseteq A_{i+j}$, for all $i \in \bar{p}$, respectively, for any given initial points $x_{0}, \bar{x}_{0} \in A_{j}$ for any given $j \in \bar{p}$. The following results hold.

(1) From (26) and by taking $\{\bar{x}\}_{n} \equiv\left\{x_{n}\right\}$ and $\left\{d\left(x_{p(n+1)+i+j}, x_{p n+i+j}\right)\right\} \rightarrow 0$, for all $i \in \bar{p}$ for $x_{0} \in A_{j}$ for any given $j \in \bar{p}$, one gets $\left\{x_{p n+i+j}\right\} \rightarrow x_{i}^{*}$, since $A_{0 i}$ is closed, for all $i \in \bar{p}$ and, from (27), $d\left(x_{i}^{*}, x_{i+1}^{*}\right)=D_{A}$, for all $i \in \bar{p}$.

(2) Again, from (26) $\left\{d\left(\bar{x}_{p(n+1)+i+j}, \bar{x}_{p n+i+j}\right)\right\} \rightarrow 0$, for all $i \in \bar{p}$ for $x_{0} \in A_{j}$ for any given $j \in \bar{p},\left\{\bar{x}_{p n+i+j}\right\} \rightarrow \bar{x}_{i}^{*}$, for all $i \in \bar{p}$.

(3) Combining results (1) and (2) with (26), it follows that $\bar{x}_{i}^{*}=x_{i}^{*}$, for all $i \in \bar{p}$.

(4) Results (1)-(3) hold irrespective of the subset $A_{j}$ for $j \in \bar{p}$ where the initial conditions of the sequences belong to, so for any $x_{0}, \bar{x}_{0} \subset \bigcup_{i \in \bar{p}} A_{i}$ (see the beginning of the proof of Lemma 6). Thus, from result (3), there are unique limit points $x_{i}^{*}$ at each subset $A_{i}$ of all the sequences $\left\{x_{n}\right\} \subset$ $\bigcup_{i \in \bar{p}} A_{i}$ such that any such sequence converges to a unique limit cycle $\left\{x_{1}^{*}, x_{2}^{*}, \ldots, x_{p}^{*}\right\}$ consisting of best proximity points of adjacent subsets $A_{i}$, for all $i \in \bar{p}$.
(5) Since $B_{i}$ is closed and approximately compact with respect to $A_{i}$, for all $i \in \bar{p}$, one gets from (28) that a subsequence of $\left\{T x_{p n+i+j}\right\}$ is convergent for each $i \in \bar{p}$; say $\left\{T x_{p n_{k}+i+j}\right\} \rightarrow z_{i}^{*} \in T\left(A_{i}\right) \subseteq B_{0 i} \subset B_{i}$, for all $i \in \bar{p}$. Since $\left\{x_{p n+i+j}\right\} \rightarrow x_{i}^{*}$, all its subsequences converge to the same limit so that $\left\{x_{p n_{k}+i+j}\right\} \rightarrow x_{i}^{*}$ and then $z_{i}^{*}=T x_{i}^{*}$ is unique, since each $x_{i}^{*}$ is unique, within each $B_{i}$, for all $i \in$ $\bar{p}$ and, again, from (28), $D_{i}=d\left(x_{i+1}^{*}, T x_{i}^{*}\right)$, for all $i \in \bar{p}$ if $T: \bigcup_{i \in \bar{p}} A_{i} \rightarrow \bigcup_{i \in \bar{p}} B_{i}$ is continuous at $x_{i}^{*}$ and $D_{i}=$ $d\left(x_{i+1}^{*}, T x_{i}^{*^{+}}\right)$if $T: \bigcup_{i \in \bar{p}} A_{i} \rightarrow \bigcup_{i \in \bar{p}} B_{i}$ has a finite-jump discontinuity at $x_{i}^{*}$, then $z_{i}^{*^{+}}=T x_{i}^{*^{+}}$(see Remark 13). The result has been proved.

A further result on the existence and uniqueness of best proximity points follows for $p$-cyclic proximal contractions subject to Definitions 8-11 under Lemma 12 and whose proof is very close to that of Theorem 15.

Theorem 16. Consider a complete metric space $(X, d)$ with nonempty subsets $A_{i}, B_{i} \subset X$ and a p-cyclic mapping $T$ : $\bigcup_{i \in \bar{p}} A_{i} \rightarrow \bigcup_{i \in \bar{p}} B_{i}$ being either $G W C P I_{p}$ or $G C P I_{p}$ or $W C P I_{p}$ or $C P I_{p}$, subject to set distances $D_{i}=d\left(A_{i+1}, B_{i}\right), D_{A}=$ $d\left(A_{i}, A_{i+1}\right)$, and $D_{B}=d\left(B_{i}, B_{i+1}\right)$, for all $i \in \bar{p}$, such that $A_{0 i}$ is nonempty and closed and $B_{0 i}$ is nonempty and $A_{i}$ is approximately compact with respect to $B_{i}$ and $T\left(A_{0 i}\right) \subseteq B_{0 i}$, for all $i \in \bar{p}$. The following properties hold.

(i) $T: \bigcup_{i \in \bar{p}} A_{i} \rightarrow \bigcup_{i \in \bar{p}} B_{i}$ has a unique best proximity point $x_{i}^{*}$ at $A_{i}$ such that $d\left(x_{i}^{*}, x_{i+1}^{*}\right)=D_{A}$, for all $i \in$ $\bar{p}$, and all the sequences $\left\{x_{n}\right\} \subset \bigcup_{i \in \bar{p}} A_{i}$ converge to $a$ unique limit cycle $\left\{x_{1}^{*}, x_{2}^{*}, \ldots, x_{p}^{*}\right\}$.

(ii) Furthermore, if $T: \bigcup_{i \in \bar{p}} A_{i} \rightarrow \bigcup_{i \in \bar{p}} B_{i}$ either is continuous, respectively, has eventual finite-jump discontinuity points, then $z_{i}^{*}=T x_{i}^{*}$, for all $i \in \bar{p}$, respectively, $z_{i}^{*^{+}}=\left(T x_{i}^{*}\right)^{+}$, for all $i \in \bar{p}$, are unique best proximity points such that $d\left(T x_{i}^{*}, T x_{i+1}^{*}\right)=D_{B}$, for all $i \in \bar{p}$, respectively, $d\left(T x_{i}^{*^{+}}, T x_{i+1}^{*^{+}}\right)=D_{B}$, for all $i \in \bar{p}$, and all the sequences $\left\{x_{n}\right\} \subset \bigcup_{i \in \bar{p}} A_{i}$ converge to a unique limit cycle $\left\{T x_{1}^{*^{+}}, T x_{2}^{*^{+}}, \ldots, T x_{p}^{*^{+}}\right\}$.

Proof. Since $T\left(A_{0 i}\right) \subseteq B_{0 i}$, for all $i \in \bar{p}$, then $T\left(A_{0 i}\right)$ and $B_{0 i}$ are nonempty, since $A_{0 i}$ is nonempty, for all $i \epsilon$ $\bar{p} . B_{0 i}$ is, furthermore, closed since $A_{i}$ is approximately compact with respect to $B_{i}$. Thus, $A_{0 i}, T\left(A_{0 i}\right)$, and $B_{0 i}$ are nonempty and closed, for all $i \in \bar{p}$. Consider any sequences $\left\{x_{n p+j}\right\},\left\{\bar{x}_{n p+j}\right\} \subseteq \bigcup_{i \in \bar{p}} A_{i}$ which satisfy

$$
d\left(x_{n p+i+1}, T x_{n p+i}\right)=d\left(\bar{x}_{n p+i+1}, T \bar{x}_{n p+i}\right)=D_{i}, \quad \forall i \in \bar{p} .
$$

One gets from Lemma 12 that, since $T\left(A_{0 i}\right) \subseteq B_{0 i}$, for all $i \in$ $\bar{p}$, 


$$
\begin{gathered}
\left\{d\left(T \bar{x}_{p(n+1)+i+j}, T x_{p n+i+j}\right)\right\} \longrightarrow 0, \\
\left\{d\left(T \bar{x}_{p(n+1)+i+j+1}, T x_{p n+i+j+1}\right)\right\} \longrightarrow 0, \\
\left\{d\left(T \bar{x}_{p n+i+j}, T x_{p n+i+j+1}\right)\right\} \\
\longrightarrow D_{B}=d\left(y_{i+j}, B_{i+1}\right)=d\left(y_{i+j}, B_{0, i+1}\right) \\
=d\left(y_{i+j}, x_{p n_{k}+i+j+1}\right), \\
\left\{d\left(\bar{x}_{p n+i+j+1}, T x_{p n+i+j}\right)\right\} \\
\longrightarrow D_{i}=d\left(y_{i+j+1}, B_{i}\right)=d\left(y_{i+j+1}, T\left(A_{i}\right)\right) \\
=d\left(y_{i+j+1}, T\left(A_{0 i}\right)\right)=d\left(y_{i+j+1}, B_{0 i}\right) \\
=d\left(y_{i+j+1}, T x_{p n_{k}+i+j}\right), \quad \forall i \in \bar{p},
\end{gathered}
$$

for some $y_{i+j} \in B_{0, i+j}, y_{i+j+1} \in B_{0, i+j+1}$ since $D_{B}=$ $d\left(B_{i}, B_{i+1}\right)=d\left(B_{0 i}, B_{0, i+1}\right)$ and $D_{i}=d\left(A_{i+1}, B_{i}\right)=$ $d\left(A_{0 i+1}, B_{0 i}\right)$, for all $i \in \bar{p}$, and some subsequences

$$
\begin{gathered}
\left\{T \bar{x}_{p n_{k}+i+j+1}\right\},\left\{T x_{p n_{k}+i+j+1}\right\} \subseteq T\left(A_{0, i+j+1}\right) \subseteq B_{0, i+j+1}, \\
\left\{T \bar{x}_{p n_{k}+i+j}\right\},\left\{T x_{p n_{k}+i+j}\right\} \subseteq T\left(A_{0, i+j+1}\right) \subseteq B_{0, i+j}
\end{gathered}
$$

of the sequences $\left\{T \bar{x}_{p n+i+j+1}\right\},\left\{T x_{p n+i+j+1}\right\} \subseteq B_{i+j+1}$ and $\left\{T \bar{x}_{p n+i+j}\right\},\left\{T x_{p n+i+j}\right\} \subseteq B_{i+j}$, for all $i \in \bar{p}$, respectively, for any given initial points $x_{0}, \bar{x}_{0} \in A_{j}$ for any given $j \in \bar{p}$. The following results hold.

(6) From (31) and by taking $\{\bar{x}\}_{n} \equiv\left\{x_{n}\right\}$, $\left\{d\left(T x_{p(n+1)+i+j}, T x_{p n+i+j}\right)\right\} \rightarrow 0$, for all $i \in \bar{p}$ for $x_{0} \in A_{j}$ for any given $j \in \bar{p}$, one gets $\left\{T x_{p n+i+j}\right\} \rightarrow z_{i}^{*}$, since $B_{0 i}$ is closed, for all $i \in \bar{p}$ and, from (32), $d\left(z_{i}^{*}, z_{i+1}^{*}\right)=D_{B}$, for all $i \in \bar{p}$.

(7) Again from (31), $\left\{d\left(T \bar{x}_{p(n+1)+i+j}, T \bar{x}_{p n+i+j}\right)\right\} \rightarrow 0$, for all $i \in \bar{p}$ for $x_{0} \in A_{j}$ for any given $j \in \bar{p}$, so that $\left\{T \bar{x}_{p n+i+j}\right\} \rightarrow \bar{z}_{i}^{*}$, for all $i \in \bar{p}$.

(8) Combining results (6) and (7) with (31), it follows that $\bar{z}_{i}^{*}=z_{i}^{*}$, for all $i \in \bar{p}$.

(9) Results (6)-(9) hold irrespective of the subset $A_{j}$ for $j \in \bar{p}$ where the initial conditions of the sequences belong to then for any $x_{0}, \bar{x}_{0} \subset \bigcup_{i \in \bar{p}} A_{i}$. Thus, considering result (8), there are unique limit points $z_{i}^{*}$ at each subset $B_{i}$ of all the sequences $\left\{T x_{n}\right\} \subset \bigcup_{i \in \bar{p}} T\left(A_{i}\right)$ such that any such sequence converges to a unique limit cycle $\left\{z_{1}^{*}, z_{2}^{*}, \ldots, z_{p}^{*}\right\}$ consisting of best proximity points of adjacent subsets $A_{i}$, for all $i \in \bar{p}$.

Since $B_{i}$ is closed and approximately compact with respect to $A_{i}$, for all $i \in \bar{p}$, one gets from (33) that a subsequence of $\left\{x_{p n+i+j}\right\}$ is convergent for each $i \in \bar{p}$; say $\left\{x_{p n_{k}+i+j}\right\} \rightarrow x_{i}^{*} \in$ $A_{0 i}$, for all $i \in \bar{p}$. Since $\left\{T x_{p n+i+j}\right\} \rightarrow z_{i}^{*}$, all its subsequences converge to the same limit so that $\left\{T x_{p n_{k}+i+j}\right\} \rightarrow z_{i}^{*}$ and $x_{i}^{*} \in A_{0 i}$ fulfilling $z_{i}^{*}=T x_{i}^{*}$ which is unique. Assume not so that there are $x_{i}^{*}, \bar{x}_{i}^{*}\left(\neq x_{i}^{*}\right) \in A_{0 i}$ for some $i \in \bar{p}$ such that $z_{i}^{*}=T x_{i}^{*}=T \bar{x}_{i}^{*}$. Assume a sequence $\left\{x_{p n_{k}+i+j}\right\} \rightarrow x_{i}^{*} \in A_{0 i}$ with $x_{0} \in A_{j}$ and a sequence $\left\{x_{p n_{k}+i+j}\right\} \rightarrow \bar{x}_{i}^{*} \in A_{0 i}$ with initial point $\bar{x}_{00} \in A_{\ell}$ and some $\bar{x}_{0}=T^{\delta} \bar{x}_{00} \in A_{j}$ for some $j, \ell \in \bar{p}$ and some nonnegative integer $\delta<p$. But then $\left\{d\left(T \bar{x}_{p(n+1)+i+j}, T \bar{x}_{p n+i+j}\right)\right\}$ does not converge to zero so that $\bar{x}_{i}^{*}=x_{i}^{*} \in A_{0 i}$ is unique, for all $i \in \bar{p}$. The distance convergence properties are independent of the fact that for the initial condition $\ell$ is as equal or distinct as $j$, as discussed in Lemma 6. If and another sequence. Since each $z_{i}^{*}$ is unique, within each $A_{i}$, for all $i \in \bar{p}$ and, again, from (33), $D_{i}=$ $d\left(x_{i+1}^{*}, T x_{i}^{*}\right)$, for all $i \in \bar{p}$ if $T: \bigcup_{i \in \bar{p}} A_{i} \rightarrow \bigcup_{i \in \bar{p}} B_{i}$ is continuous at $x_{i}^{*}$ and $D_{i}=d\left(x_{i+1}^{*}, T x_{i}^{*^{+}}\right)$if $T: \bigcup_{i \in \bar{p}} A_{i} \rightarrow$ $\bigcup_{i \in \bar{p}} B_{i}$ has a finite-jump discontinuity at $x_{i}^{*}$, then $z_{i}^{*^{+}}=T x_{i}^{*^{+}}$ (see Remark 13). The result has been proved.

Example 17. Consider a 2-cyclic proximal contraction with respect to its domain: $T: A_{1} \cup A_{2} \rightarrow B_{1} \cup B_{2}$, where $A_{i}$ and $B_{i}$ for $i=1,2$ are nonempty closed subsets of $\mathbf{R}$. Take any sequences $\left\{x_{n}\right\} \subset A_{1},\left\{y_{n}\right\} \subset A_{2}$ being subsequences of $\left\{z_{n}\right\} \subset A_{1} \cup A_{2}$ defined either by $z_{2 n}=x_{n}, z_{2 n+1}=y_{n}$ or by $z_{2 n}=y_{n}, z_{2 n+1}=x_{n}$, for all $n \in \mathbf{Z}_{0+}$, and subject to the constraints below under the Euclidean metric $d(x, y)=$ $|x-y|$, for all $x, y \in \mathbf{R}$ for some contractive real constant $\alpha \epsilon$ $[0,1)$ such that $\alpha_{1}=\alpha_{2}=\alpha$ and $(\mathbf{R}, d)$ is a complete metric space and also a Banach space. Assume that $A_{1}=\left[a_{1}, \bar{a}_{1}\right]$ and $A_{2}=\left[a_{2}, \bar{a}_{2}\right]$ with $\bar{a}_{1}<0$ and $a_{2}>0$ and $B_{1}=\left[\bar{b}_{1}, \bar{b}_{1}\right]$ and $B_{2}=\left[\underline{b_{2}}, \bar{b}_{2}\right]$ with $\bar{a}_{1} \leq 0$ and $\underline{a_{2}} \geq 0, \underline{b_{1}} \leq \underline{a_{1}}, 0 \geq \bar{b}_{1} \geq \bar{a}_{1}$, $0 \leq b_{2} \leq a_{2}$, and $\bar{b}_{2} \geq \bar{a}_{2}$, so that $T\left(\overline{A_{1}}\right) \subseteq \bar{B}_{1}, T\left(A_{2}\right) \subseteq B_{2}$ with

$$
\begin{aligned}
& D_{A}=d\left(A_{1}, A_{2}\right)=\left|\overline{a_{1}}\right|+\underline{a_{2}}, \\
& D_{B}=d\left(B_{1}, B_{2}\right)=\left|\overline{b_{1}}\right|+\underline{b_{2}}, \\
& D_{1}=d\left(A_{2}, B_{1}\right)=\underline{a_{2}}+\left|\bar{b}_{1}\right|, \\
& D_{2}=d\left(A_{1}, B_{2}\right)=\left|\bar{a}_{1}\right|+\underline{b_{2}},
\end{aligned}
$$

and $T: A_{1} \cup A_{2} \rightarrow B_{1} \cup B_{2}$ is a $\mathrm{CPD}_{2}$ (Definition 2 with $p=2$ ) if the subsequent constraints hold for all $n \in \mathbf{Z}_{0+}$ :

$$
\begin{aligned}
& \quad y_{n+1}+\left|T x_{n}\right|=D_{1}, \quad T y_{n}+\left|x_{n+1}\right|=D_{2} \\
& \left|x_{n+2}-x_{n+1}\right| \\
& =|| x_{n+1}|-| x_{n+2}||=\left|T y_{n}-T y_{n+1}\right| \leq \alpha\left|x_{n+1}-x_{n}\right| \\
& \left|y_{n+2}-y_{n+1}\right|=|| T x_{n}|-| T x_{n+1}|| \leq \alpha\left|y_{n+1}-y_{n}\right| \\
& \quad y_{n+1}-x_{n+1} \mid \\
& \quad=y_{n+1}+\left|x_{n+1}\right|=D_{1}+D_{2}-\left|T x_{n}\right|-T y_{n} \\
& \leq \alpha\left|y_{n}-x_{n}\right|+(1-\alpha) D_{A} \\
& \leq \alpha\left|y_{n}-x_{n}\right|+(1-\alpha) D_{A} \\
& \quad=\alpha\left(y_{n}+\left|x_{n}\right|\right)+(1-\alpha) D_{A} .
\end{aligned}
$$


In particular, (37)-(40a) and (40b) are satisfied if, for all $n \in$ $\mathbf{Z}_{0+}$,

$$
\begin{gathered}
T y_{n} \geq D_{2}-\alpha y_{n}-\frac{1-\alpha}{2} D_{A}, \\
T x_{n} \leq-D_{1}-\alpha x_{n}+\frac{1-\alpha}{2} D_{A} \\
\left(\text { equivalently, } D_{1}-\alpha\left|x_{n}\right|-\frac{1-\alpha}{2} D_{A} \leq\left|T x_{n}\right|\right), \\
y_{n+1} \leq \alpha\left|x_{n}\right|-\frac{1-\alpha}{2} D_{A}, \\
\left|x_{n+1}\right| \leq \alpha y_{n}+\frac{1-\alpha}{2} D_{A} .
\end{gathered}
$$

Parallel results for the case when $T: A_{1} \cup A_{2} \rightarrow B_{1} \cup B_{2}$

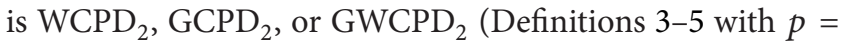
2 ) can be discussed in the same way with the appropriate extensions for the contractive constant or function. It follows that $\left\{d\left(y_{n}, y_{n+1}\right)\right\} \rightarrow 0,\left\{d\left(x_{n}, x_{n+1}\right)\right\} \rightarrow 0,\left\{d\left(z_{n}, z_{n+1}\right)\right\} \rightarrow$ $D_{A},\left\{y_{n}\right\} \rightarrow a_{2},\left\{x_{n}\right\} \rightarrow \bar{a}_{1},\left\{d\left(T y_{n}, T y_{n+1}\right)\right\} \rightarrow 0,\left\{d\left(T x_{n}\right.\right.$, $\left.\left.T x_{n+1}\right)\right\} \rightarrow 0$ and, according to (40a), (40b), and (35)-(36), $\left\{d\left(T z_{n}, T z_{n+1}\right)\right\} \rightarrow D_{B}$, since $\left\{\left|T\left(y_{n}-\left|x_{n}\right|\right)\right|\right\} \rightarrow D_{B}=$ $D_{1}+D_{2}-D_{A},\left\{T y_{n}\right\} \rightarrow T \underline{a}_{2}=\underline{b}_{2}$, and $\left\{T x_{n}\right\} \rightarrow T \bar{a}_{1}=\bar{b}_{1}$.

Example 18. Consider Example 17 in the case that $T: A_{1} \cup$ $A_{2} \rightarrow B_{1} \cup B_{2}$ is $\mathrm{CPI}_{2}, \mathrm{WCPI}_{2}, \mathrm{GCPI}_{2}$, or $\mathrm{GWCPI}_{2}$ (Definitions 8-11 with $p=2$ ); (40a) using (37) can be reformulated accordingly. In particular, if it is $\mathrm{CPI}_{2}$, then one gets for some real constant $\beta \in[0,1)$

$$
\begin{aligned}
& \left|T x_{n+1}\right|+T y_{n+1} \\
& \quad=T y_{n+1}-T x_{n+1}=D_{1}+D_{2}-\left|x_{n+2}\right|-y_{n+2} \\
& \quad=D_{A}+D_{B}-\left|x_{n+2}\right|-y_{n+2} \\
& \quad \leq \beta\left(\left|x_{n}\right|+y_{n}\right)+(1-\beta) D_{B}, \quad \forall n \in \mathbf{Z}_{0+} .
\end{aligned}
$$

Then, $\left\{\left|T\left(y_{n}-\left|x_{n}\right|\right)\right|\right\} \equiv\left\{d\left(T z_{n}, T z_{n+1}\right)\right\} \rightarrow D_{B}=D_{1}+D_{2}-$ $D_{A},\left\{\left|x_{n}\right|+y_{n}\right\} \equiv\left\{d\left(z_{n}, z_{n+1}\right)\right\} \rightarrow D_{A},\left\{d\left(y_{n}, y_{n+1}\right)\right\} \rightarrow 0$, $\left\{d\left(x_{n}, x_{n+1}\right)\right\} \rightarrow 0,\left\{T y_{n}\right\} \rightarrow T a_{2}=\underline{b_{2}},\left\{T x_{n}\right\} \rightarrow T \bar{a}_{1}=\bar{b}_{1}$, $\left\{y_{n}\right\} \rightarrow a_{2}$, and $\left\{x_{n}\right\} \rightarrow \bar{a}_{1}$.

\section{Conflict of Interests}

The authors declare that there is no conflict of interests regarding the publication of this paper.

\section{Acknowledgments}

The authors are very grateful to the Spanish Government for its support of this research trough Grant DPI2012-30651 and to the Basque Government for its support of this research through Grants IT378-10 and SAIOTEK S-PE12UN015. They are also grateful to the University of Basque Country for its financial support through Grant UFI 2011/07. Finally, they thank the reviewers for their useful suggestions towards the improvement of the first version of this paper.

\section{References}

[1] S. Sadiq Basha, "Best proximity points: optimal solutions," Journal of Optimization Theory and Applications, vol. 151, no. 1, pp. 210-216, 2011.

[2] S. Karpagam and S. Agrawal, "Best proximity point theorems for $p$-cyclic Meir-Keeler contractions," Fixed Point Theory and Applications, vol. 2009, Article ID 197308, 9 pages, 2009.

[3] A. A. Eldred and P. Veeramani, "Existence and convergence of best proximity points," Journal of Mathematical Analysis and Applications, vol. 323, no. 2, pp. 1001-1006, 2006.

[4] M. Gabeleh, "Best proximity points for weak proximal contractions," Bulletin of the Malaysian Mathematical Society. In press.

[5] B. Samet, "Some results on best proximity points," Journal of Optimization Theory and Applications, vol. 159, no. 1, pp. 281291, 2013

[6] M. Jleli and B. Samet, "Best proximity points for $\alpha-\psi$-proximal contractive type mappings and applications," Bulletin des Sciences Mathematiques, vol. 137, no. 8, pp. 977-995, 2013.

[7] M. de la Sen, "Linking contractive self-mappings and cyclic Meir-Keeler contractions with Kannan self-mappings," Fixed Point Theory and Applications, vol. 2010, Article ID 572057, 2010.

[8] C.-M. Chen and C. H. Lin, "Best periodic proximity points for cyclic weaker Meir-Keeler contractions," Journal of Applied Mathematics, vol. 2012, Article ID 782389, 7 pages, 2012.

[9] M. de la Sen, "Some combined relations between contractive mappings, Kannan mappings, reasonable expansive mappings, and T-stability," Fixed Point Theory and Applications, vol. 2009, Article ID 815637, 2009.

[10] M. de la Sen and R. P. Agarwal, "Some fixed point-type results for a class of extended cyclic self-mappings with a more general contractive condition," Fixed Point Theory and Applications, vol. 2011, article 59, 2011.

[11] M. de la Sen, R. P. Agarwal, and R. Nistal, "Non-expansive and potentially expansive properties of two modified p-cyclic self-maps in metric spaces," Journal of Nonlinear and Convex Analysis, vol. 14, no. 4, pp. 661-686, 2013.

[12] M. De la Sen and R. P. Agarwal, "Fixed point-type results for a class of extended cyclic self-mappings under three general weak contractive conditions of rational type," Fixed Point Theory and Applications, vol. 2011, article 102, 2011.

[13] N. Hussain, "Common fixed points in best approximation for Banach operator pairs with Ćirić type I-contractions," Journal of Mathematical Analysis and Applications, vol. 338, no. 2, pp. 1351-1363, 2008.

[14] C. Mongkolkeha, Y. J. Cho, and P. Kumam, "Best proximity points for generalized proximal $C$-contraction mappings in metric spaces with partial orders," Journal of Inequalities and Applications, vol. 2013, article 94, 2013.

[15] S. S. Basha and N. Shahzad, "Best proximity point theorems for generalized proximal contractions," Fixed Point Theory and Applications, vol. 2012, article 42, 2012.

[16] V. M. Marchenko and J. J. Luazo, "On the stability of hybrid differential-difference systems," Differential Equations, vol. 45, no. 5, pp. 743-756, 2009.

[17] V. M. Marchenko, "Hybrid discrete-continuous systems: II. Controllability and reachability," Differential Equations, vol. 49, no. 1, pp. 112-125, 2013.

[18] V. M. Marchenko, "Observability of hybrid discrete-continuous systems," Differential Equations, vol. 49, no. 11, pp. 1389-1404, 2013. 
[19] A. Bilbao-Guillerna, M. de la Sen, A. Ibeas, and S. AlonsoQuesada, "Robustly stable multiestimation scheme for adaptive control and identification with model reduction issues," Discrete Dynamics in Nature and Society, vol. 2005, no. 1, pp. 31-67, 2005.

[20] S. Alonso-Quesada and M. De la Sen, "Robust adaptive control of discrete nominally stabilizable plants," Applied Mathematics and Computation, vol. 150, no. 2, pp. 555-583, 2004.

[21] M. de la Sen, "Adaptive stabilization of first-order systems using estimates modification based on a Sylvester determinant test," Computers and Mathematics with Applications, vol. 37, no. 10, pp. 51-62, 1999.

[22] M. De la Sen, "About robust stability of dynamic systems with time delays through fixed point theory," Fixed Point Theory and Applications, vol. 2008, Article ID 480187, 2008.

[23] S. N. Mishra, S. L. Singh, and R. Pant, "Some new results on stability of fixed points," Chaos, Solitons \& Fractals, vol. 45, no. 7, pp. 1012-1016, 2012.

[24] M. De la Sen, "About robust stability of Caputo linear fractional dynamic systems with time delays through fixed point theory," Fixed Point Theory and Applications, vol. 2011, Article ID 867932, 19 pages, 2011.

[25] M. de la Sen, "Preserving positive realness through discretization," Positivity, vol. 6, no. 1, pp. 31-45, 2002.

[26] M. de la Sen and N. Luo, "Design of linear observers for a class of linear hybrid systems," International Journal of Systems Science, vol. 31, no. 9, pp. 1077-1090, 2000.

[27] H. K. Pathak and M. S. Khan, "Common fixed point results and its applications to best approximation in ordered semi-convex structure," Bulletin of Mathematical Analysis and Applications, vol. 1, no. 1, pp. 30-48, 2009.

[28] H. K. Nashine and M. S. Khan, "An application of fixed point theorem to best approximation in locally convex space," Applied Mathematics Letters, vol. 23, no. 2, pp. 121-127, 2010.

[29] H. A. Kenary, H. Rezaei, Y. Gheisari, and C. Park, "On the stability of set-valued functional equations with the fixed point alternative," Fixed Point Theory and Applications, vol. 2012, article 81, 2012.

[30] T. Z. Xu and J. M. Rassias, "A fixed point approach to the stability of an AQ-functional equation on $\beta$-Banach modules," Fixed Point Theory and Applications, 2012:32, 14 pages, 2012.

[31] W. Shatanawi, "Best proximity point on nonlinear contractive condition," Journal of Physics: Conference Series, vol. 435, no. 1, Article ID 012006, 2013.

[32] M. Gabeleh, "Best proximity points theorems via proximal nonself mappings," Journal of Optimization Theory and Applications, 2014.

[33] N. Hussain, A. Latif, and P. Salimi, "Best proximity point results for modified Suzuki $\alpha-\psi$-proximal contractions," Fixed Point Theory and Applications, vol. 2014, article 10, 2014.

[34] W. Sanhan, C. Mongkolkeha, and P. Kumam, "Generalized proximal $\psi$-contraction mappings and best proximity points," Abstract and Applied Analysis, vol. 2012, Article ID 896912, 19 pages, 2012.

[35] W. Sintunavarat and P. Kumam, "The existence theorems of an optimal approximate solution for generalized proximal contraction mappings," Abstract and Applied Analysis, vol. 2013, Article ID 375604, 8 pages, 2013. 


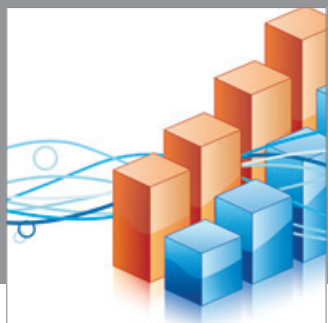

Advances in

Operations Research

mansans

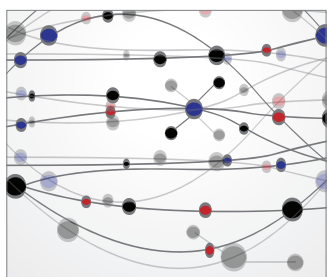

The Scientific World Journal
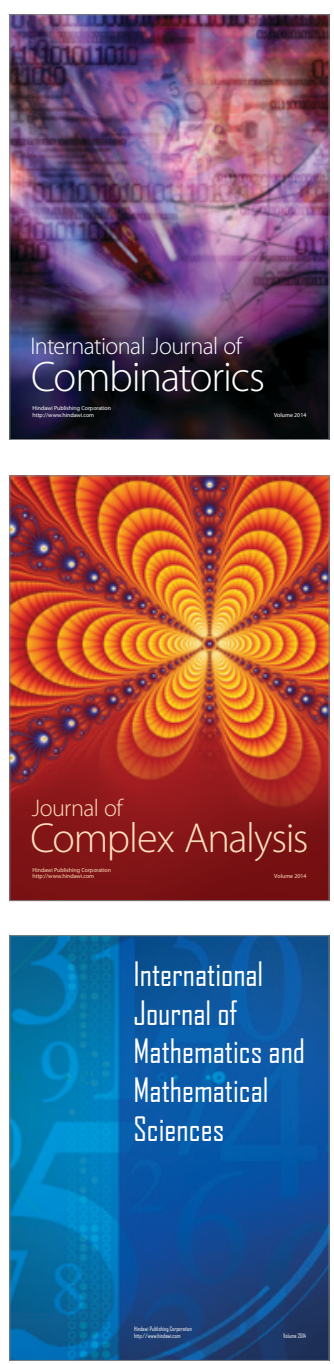
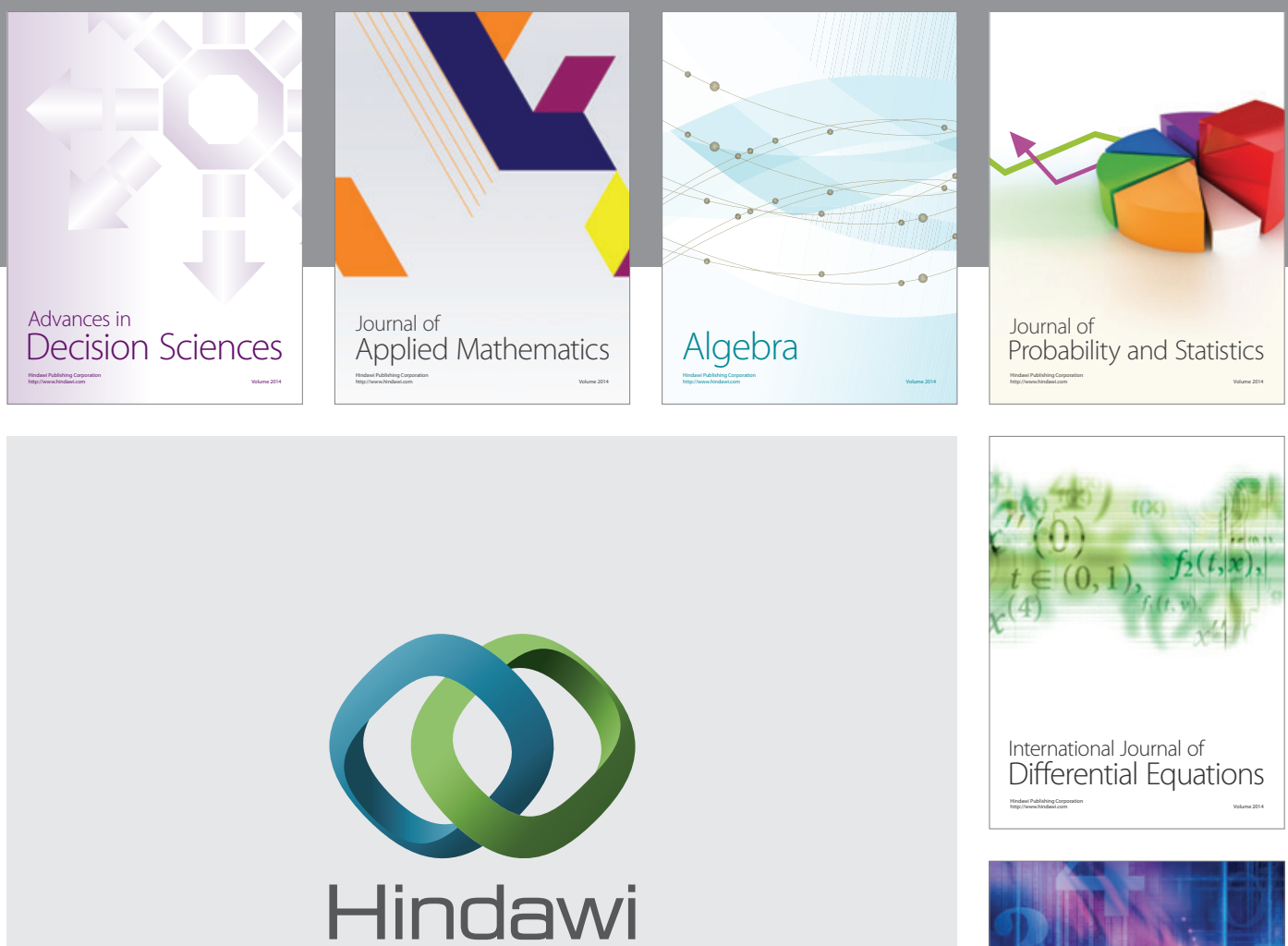

Submit your manuscripts at http://www.hindawi.com
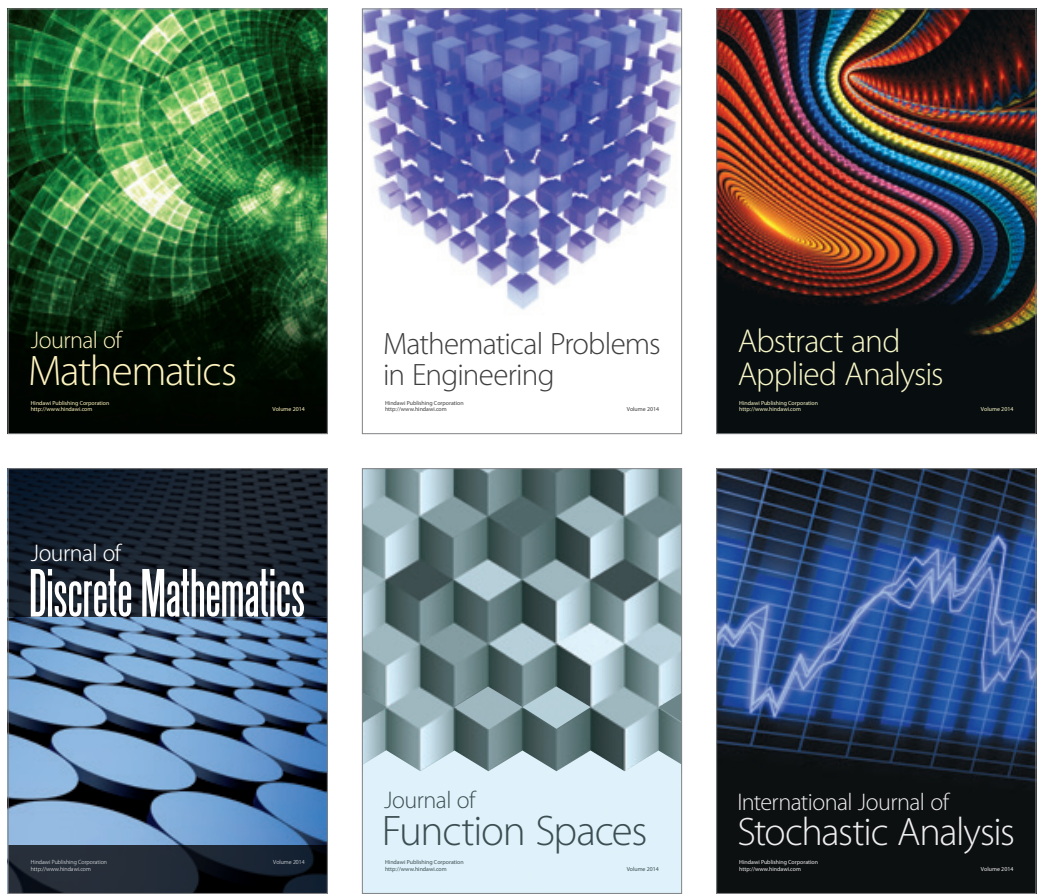

Journal of

Function Spaces

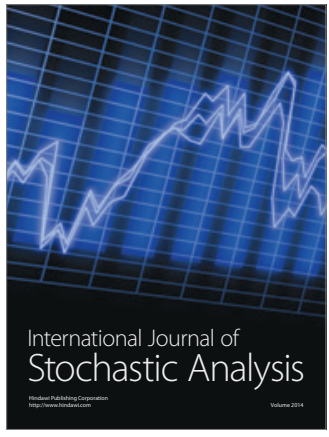

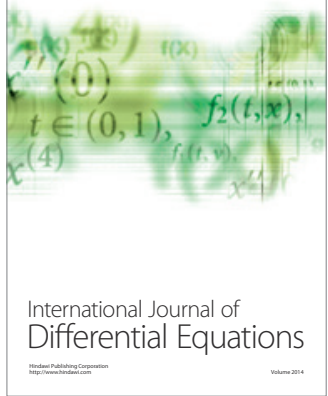
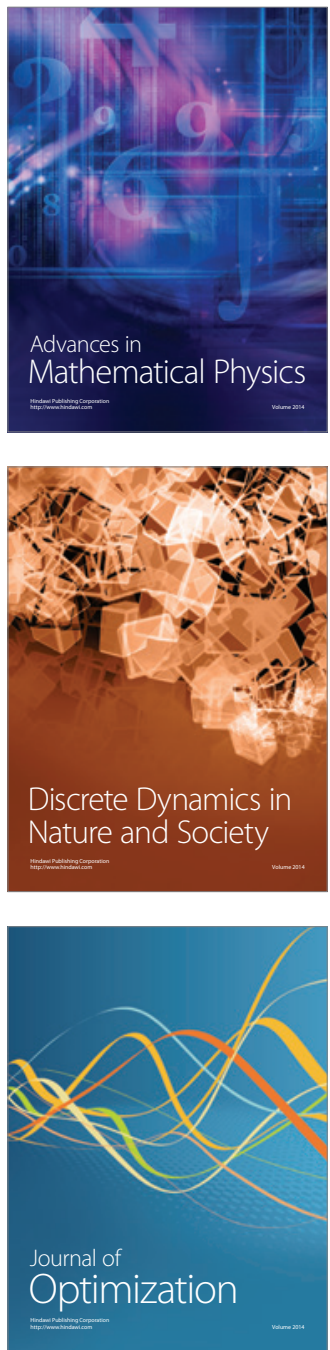